\title{
Long Range Scattering for the Maxwell-Schrödinger System with Large Magnetic Field Data and Small Schrödinger Data ${ }^{\dagger}$
}

\author{
By \\ Jean GiniBRE* and Giorgio Velo**
}

\begin{abstract}
We study the theory of scattering for the Maxwell-Schrödinger system in the Coulomb gauge in space dimension 3. We prove in particular the existence of modified wave operators for that system with no size restriction on the magnetic field data in the framework of a direct method which requires smallness of the Schrödinger data, and we determine the asymptotic behaviour in time of solutions in the range of the wave operators.
\end{abstract}

\section{$\S 1 . \quad$ Introduction}

This paper is devoted to the theory of scattering and more precisely to the construction of modified wave operators for the Maxwell-Schrödinger system $(\mathrm{MS})_{3}$ in $3+1$ dimensional space time. That system describes the evolution of a charged nonrelativistic quantum mechanical particle interacting with the (classical) electromagnetic field it generates. It can be written as follows:

$$
\left\{\begin{array}{l}
i \partial_{t} u=-(1 / 2) \Delta_{A} u+A_{0} u \\
\square A_{0}-\partial_{t}\left(\partial_{t} A_{0}+\nabla \cdot A\right)=|u|^{2} \\
\square A+\nabla\left(\partial_{t} A_{0}+\nabla \cdot A\right)=\operatorname{Im} \bar{u} \nabla_{A} u .
\end{array}\right.
$$

Communicated by T. Kawai. Received October 14, 2004.

2000 Mathematics Subject Classification(s): 35P25, 35B40, 35Q40, 81U99.

Key words: Long range scattering, modified wave operators, Maxwell-Schrödinger system

${ }^{\dagger}$ Work supported in part by NATO Collaborative Linkage Grant 979341.

* Laboratoire de Physique Théorique (Unité Mixte de Recherche (CNRS) UMR 8627), Université de Paris XI, Bâtiment 210, F-91405 ORSAY Cedex, France.

e-mail: jean.ginibre@th.u-psud.fr

**Dipartimento di Fisica, Università di Bologna, and INFN, Sezione di Bologna, Italy. e-mail: velo@bo.infn.it 
Here $u$ and $\left(A, A_{0}\right)$ are respectively a complex valued function and an $\mathbb{R}^{3+1}$ valued function defined in space time $\mathbb{R}^{3+1}, \nabla_{A}=\nabla-i A, \Delta_{A}=\nabla_{A}^{2}$ and $\square=\partial_{t}^{2}-\Delta$ is the d'Alembertian in $\mathbb{R}^{3+1}$. We shall consider that system exclusively in the Coulomb gauge $\nabla \cdot A=0$. In that gauge, one can replace the system (1.1) by a formally equivalent one in the following standard way. The second equation of (1.1) can be solved for $A_{0}$ by

$$
A_{0}=-\Delta^{-1}|u|^{2}=(4 \pi|x|)^{-1} *|u|^{2} \equiv g\left(|u|^{2}\right)
$$

Substituting (1.2) into the first and last equations of (1.1) yields the new system

$$
\left\{\begin{array}{l}
i \partial_{t} u=-(1 / 2) \Delta_{A} u+g\left(|u|^{2}\right) u \\
\square A=P \operatorname{Im} \bar{u} \nabla_{A} u
\end{array}\right.
$$

where $P=\mathbb{1}-\nabla \Delta^{-1} \nabla$ is the projector on divergence free vector fields, together with the Coulomb gauge condition $\nabla \cdot A=0$ which is formally preserved by the evolution. From now on we restrict our attention to the system (1.3).

The (MS) $)_{3}$ system is known to be locally well posed in sufficiently regular spaces [11], [12] and to have global weak solutions in the energy space [9] in various gauges including the Coulomb gauge. However that system is so far not known to be globally well posed in any space.

A large amount of work has been devoted to the theory of scattering for nonlinear equations and systems centering on the Schrödinger equation, in particular for nonlinear Schrödinger (NLS) equations, Hartree equations, KleinGordon-Schrödinger (KGS), Wave-Schrödinger (WS) and Maxwell-Schrödinger (MS) systems. As in the case of the linear Schrödinger equation, one must distinguish the short range case from the long range case. In the former case, ordinary wave operators are expected and in a number of cases proved to exist, describing solutions where the Schrödinger function behaves asymptotically like a solution of the free Schrödinger equation. In the latter case, ordinary wave operators do not exist and have to be replaced by modified wave operators including a suitable phase in their definition. In that respect, the (MS) $)_{3}$ system (1.1) belongs to the borderline (Coulomb) long range case, because of the $t^{-1}$ decay in $L^{\infty}$ norm of solutions of the wave equation. Such is the case also for the Hartree equation with $|x|^{-1}$ potential, for the Wave-Schrödinger system $(\mathrm{WS})_{3}$ in $\mathbb{R}^{3+1}$ and for the Klein-Gordon-Schrödinger system (KGS) $)_{2}$ in $\mathbb{R}^{2+1}$.

The construction of modified wave operators for the previous long range equations and systems has been tackled by two methods. The first one was initiated in [13] on the example of the NLS equation in $\mathbb{R}^{1+1}$ and subsequently applied to the NLS equation in $\mathbb{R}^{2+1}$ and $\mathbb{R}^{3+1}$ and to the Hartree equation [1], 
to the $(\mathrm{KGS})_{2}$ system [14], [15], [16], [17], to the (WS) $)_{3}$ system [18] and to the $(\mathrm{MS})_{3}$ system [19], [21]. That method is rather direct, starting from the original equation or system. It will be sketched below. It is restricted to the (Coulomb) limiting long range case, and requires a smallness condition on the asymptotic state of the Schrödinger function. Early applications of the method required in addition a support condition on the Fourier transform of the Schrödinger asymptotic state and a smallness condition of the Klein-Gordon or Maxwell field in the case of the $(\mathrm{KGS})_{2}$ or $(\mathrm{MS})_{3}$ system respectively [14], [21]. The support condition was subsequently removed for the (KGS) $)_{2}$ and (MS $)_{3}$ system and the method was applied to the (WS $)_{3}$ system without a support condition, at the expense of adding a correction term to the Schrödinger asymptotic function [15], [18], [19]. The smallness condition of the KG field was then removed for the (KGS $)_{2}$ system, first with and then without a support condition [16], [17]. Finally the smallness condition on the wave field was removed for the (WS $)_{3}$ system, without a support condition or a correction term to the Schrödinger asymptotic function [8].

In the present paper, we extend the results of our previous paper [8] from the (WS $)_{3}$ system to the $(\mathrm{MS})_{3}$ system in the Coulomb gauge (1.3). In particular we prove the existence of modified wave operators without any smallness condition on the magnetic potential $A$, and without a support condition or a correction term on the asymptotic Schrödinger function. In addition, in the same spirit as in [8], we treat the problem in function spaces that are as large as possible, namely with regularity as low as possible. As a consequence, we require only a much lower regularity of the asymptotic state than in previous works.

For completeness and although we shall not make use of that fact in the present paper, we mention that the same problem for the Hartree equation and for the (WS $)_{3}$ and $(\mathrm{MS})_{3}$ system can also be treated by a more complex method where one first applies a phase-amplitude separation to the Schrödinger function. The main interest of that method is to remove the smallness condition on the Schrödinger function, and to go beyond the Coulomb limiting case for the Hartree equation. That method has been applied in particular to the (WS $)_{3}$ system and to the $(\mathrm{MS})_{3}$ system in a special case [4], [5], [6].

We now sketch briefly the method of construction of the modified wave operators initiated in [13]. That construction basically consists in solving the Cauchy problem for the system (1.3) with infinite initial time, namely in constructing solutions $(u, A)$ with prescribed asymptotic behaviour at infinity in time. We restrict our attention to time going to $+\infty$. That asymptotic 
behaviour is imposed in the form of suitable approximate solutions $\left(u_{a}, A_{a}\right)$ of the system (1.3). The approximate solutions are parametrized by data $\left(u_{+}, A_{+}, \dot{A}_{+}\right)$which play the role of (actually would be in simpler e.g. short range cases) initial data at time zero for a simpler evolution. One then looks for exact solutions $(u, A)$ of the system (1.3), the difference of which with the given asymptotic ones tends to zero at infinity in time in a suitable sense, more precisely, in suitable norms. The wave operator is then defined traditionally as the map $\Omega_{+}:\left(u_{+}, A_{+}, \dot{A}_{+}\right) \rightarrow\left(u, A, \partial_{t} A\right)(0)$. However what really matters is the solution $(u, A)$ in the neighbourhood of infinity in time, namely in some interval $[T, \infty)$, and we shall restrict our attention to the construction of such solutions. Continuing such solutions down to $t=0$ is a somewhat different question, connected with the global Cauchy problem at finite times, which we shall not touch here, especially since the $(\mathrm{MS})_{3}$ system is not known to be globally well posed in any function space.

The construction of solutions $(u, A)$ with prescribed asymptotic behaviour $\left(u_{a}, A_{a}\right)$ is performed in two steps.

$\underline{\text { Step 1. }}$. One looks for $(u, A)$ in the form $(u, A)=\left(u_{a}+v, A_{a}+B\right)$ with $\nabla \cdot A_{a}=$ $\nabla \cdot B=0$. The system satisfied by the new functions $(v, B)$ can be written as

$$
\left\{\begin{array}{l}
i \partial_{t} v=-(1 / 2) \Delta_{A} v+g\left(|u|^{2}\right) v+G_{1}-R_{1} \\
\square B=G_{2}-R_{2}
\end{array}\right.
$$

where $G_{1}$ and $G_{2}$ are defined by

$$
\left\{\begin{array}{l}
G_{1}=i B \cdot \nabla_{A_{a}} u_{a}+(1 / 2) B^{2} u_{a}+g\left(|v|^{2}+2 \operatorname{Re} \bar{u}_{a} v\right) u_{a} \\
G_{2}=P \operatorname{Im}\left(\bar{v} \nabla_{A} v+2 \bar{v} \nabla_{A} u_{a}\right)-P B\left|u_{a}\right|^{2}
\end{array}\right.
$$

and the remainders are defined by

$$
\left\{\begin{array}{l}
R_{1}=i \partial_{t} u_{a}+(1 / 2) \Delta_{A_{a}} u_{a}-g\left(\left|u_{a}\right|^{2}\right) u_{a} \\
R_{2}=\square A_{a}-P \operatorname{Im} \bar{u}_{a} \nabla_{A_{a}} u_{a} .
\end{array}\right.
$$

It is technically useful to consider also the partly linearized system for functions $\left(v^{\prime}, B^{\prime}\right)$

$$
\left\{\begin{array}{l}
i \partial_{t} v^{\prime}=-(1 / 2) \Delta_{A} v^{\prime}+g\left(|u|^{2}\right) v^{\prime}+G_{1}-R_{1} \\
\square B^{\prime}=G_{2}-R_{2} .
\end{array}\right.
$$

The first step of the method consists in solving the system $(1.4)$ for $(v, B)$, with $(v, B)$ tending to zero at infinity in time in suitable norms, under assumptions 
on $\left(u_{a}, A_{a}\right)$ of a general nature, the most important of which being decay assumptions on the remainders $R_{1}$ and $R_{2}$. That can be done as follows. One first solves the linearized system $(1.7)$ for $\left(v^{\prime}, B^{\prime}\right)$ with given $(v, B)$ and initial data $\left(v^{\prime}, B^{\prime}\right)\left(t_{0}\right)=0$ for some large finite $t_{0}$. One then takes the limit $t_{0} \rightarrow \infty$ of that solution, thereby obtaining a solution $\left(v^{\prime}, B^{\prime}\right)$ of $(1.7)$ which tends to zero at infinity in time. That construction defines a map $\phi:(v, B) \rightarrow\left(v^{\prime}, B^{\prime}\right)$. One then shows by a contraction method that the map $\phi$ has a fixed point. That first step will be performed in Section 2 .

Step 2. The second step of the method consists in constructing approximate asymptotic solutions $\left(u_{a}, A_{a}\right)$ satisfying the general estimates needed to perform Step 1. With the weak time decay allowed by our treatment of Step 1, one can take the simplest version of the asymptotic form used in previous works [6], [19], [21]. Thus we choose

$$
u_{a}=M D \exp (-i \varphi) w_{+}
$$

where

$$
\begin{aligned}
M & \equiv M(t)=\exp \left(i x^{2} / 2 t\right), \\
D(t) & =(i t)^{-n / 2} D_{0}(t), \quad\left(D_{0}(t) f\right)(x)=f(x / t),
\end{aligned}
$$

$\varphi$ is a real phase to be chosen below and $w_{+}=F u_{+}$. We furthermore choose $A_{a}$ in the form $A_{a}=A_{0}+A_{1}$ where $A_{0}$ is the solution of the free wave equation $\square A_{0}=0$ given by

$$
A_{0}=\cos \omega t A_{+}+\omega^{-1} \sin \omega t \dot{A}_{+}
$$

where $\omega=(-\Delta)^{1 / 2}$, and where

$$
A_{1}(t)=\int_{t}^{\infty} d t^{\prime}\left(\omega t^{\prime}\right)^{-1} \sin \left(\omega\left(t^{\prime}-t\right)\right) P x\left|u_{a}\left(t^{\prime}\right)\right|^{2} .
$$

Substituting (1.8) into (1.12) yields

$$
A_{1}(t)=t^{-1} D_{0}(t) \widetilde{A}_{1}
$$

where

$$
\widetilde{A}_{1}=\int_{1}^{\infty} d \nu \nu^{-3} \omega^{-1} \sin (\omega(\nu-1)) D_{0}(\nu) P x\left|w_{+}\right|^{2} .
$$

In particular $\widetilde{A}_{1}$ is constant in time. We finally choose $\varphi$ by imposing

$$
\varphi(1)=0, \quad \partial_{t} \varphi=t^{-1}\left(g\left(\left|w_{+}\right|^{2}\right)-x \cdot \widetilde{A}_{1}\right)
$$


so that

$$
\varphi=(\ln t)\left(g\left(\left|w_{+}\right|^{2}\right)-x \cdot \widetilde{A}_{1}\right) .
$$

We shall show in Section 3 that the previous choice fulfils the conditions needed for Step 1, under suitable assumptions on the asymptotic state $\left(u_{+}, A_{+}, \dot{A}_{+}\right)$.

In order to state our results we introduce some notation. We denote by $F$ the Fourier transform, by $\langle\cdot, \cdot\rangle$ the scalar product in $L^{2}$ and by $\|\cdot\|_{r}$ the norm in $L^{r} \equiv L^{r}\left(\mathbb{R}^{3}\right), 1 \leq r \leq \infty$ and we define $\delta(r)=3 / 2-3 / r$. For any nonnegative integer $k$ and for $1 \leq r \leq \infty$, we denote by $W_{r}^{k}$ the Sobolev spaces

$$
W_{r}^{k}=\left\{u:\left\|u ; W_{r}^{k}\right\|=\sum_{\alpha: 0 \leq|\alpha| \leq k}\left\|\partial_{x}^{\alpha} u\right\|_{r}<\infty\right\}
$$

where $\alpha$ is a multiindex, so that $H^{k}=W_{2}^{k}$. We shall need the weighted Sobolev spaces $H^{k, s}$ defined for $k, s \in \mathbb{R}$ by

$$
H^{k, s}=\left\{u:\left\|u ; H^{k, s}\right\|=\left\|\left(1+x^{2}\right)^{s / 2}(1-\Delta)^{k / 2} u\right\|_{2}<\infty\right\}
$$

so that $H^{k}=H^{k, 0}$. For any interval $I$, for any Banach space $X$ and for any $q$, $1 \leq q \leq \infty$, we denote by $L^{q}(I, X)$ (resp. $\left.L_{l o c}^{q}(I, X)\right)$ the space of $L^{q}$ integrable (resp. locally $L^{q}$ integrable) functions from $I$ to $X$ if $q<\infty$ and the space of measurable essentially bounded (resp. locally essentially bounded) functions from $I$ to $X$ if $q=\infty$. For any $h \in \mathcal{C}\left([1, \infty), \mathbb{R}^{+}\right)$, non increasing and tending to zero at infinity and for any interval $I \subset[1, \infty)$, we define the space

$$
X(I)=\left\{(v, B): v \in \mathcal{C}\left(I, H^{2}\right) \cap \mathcal{C}^{1}\left(I, L^{2}\right),\right.
$$

$$
\begin{gathered}
\|(v, B) ; X(I)\| \equiv \operatorname{Sup}_{t \in I} h(t)^{-1}\left(\left\|v(t) ; H^{2}\right\|+\left\|\partial_{t} v(t)\right\|_{2}+\left\|v ; L^{8 / 3}\left(J, W_{4}^{1}\right)\right\|\right. \\
\left.\left.+\left\|B ; L^{4}\left(J, W_{4}^{1}\right)\right\|+\left\|\partial_{t} B ; L^{4}\left(J, L^{4}\right)\right\|\right)<\infty\right\}
\end{gathered}
$$

where $J=[t, \infty) \cap I$.

We can now state our result.

Proposition 1.1. Let $h(t)=t^{-1}(2+\ell n t)^{2}$ and let $X(\cdot)$ be defined by (1.17). Let $u_{a}$ be defined by (1.8) with $w_{+}=F u_{+}$and with $\varphi$ defined by (1.16) (1.2) (1.14). Let $A_{a}=A_{0}+A_{1}$ with $A_{0}$ defined by (1.11) and $A_{1}$ by (1.13) (1.14). Let $u_{+} \in H^{3,1} \cap H^{1,3}$ with $\left\|x w_{+}\right\|_{4}$ and $\left\|w_{+}\right\|_{3}$ sufficiently small. Let $\nabla^{2} A_{+}, \nabla \dot{A}_{+}, \nabla^{2}\left(x \cdot A_{+}\right)$and $\nabla\left(x \cdot \dot{A}_{+}\right) \in W_{1}^{1}$ with $A_{+}, x \cdot A_{+} \in L^{3}$ and $\dot{A}_{+}$, $x \cdot \dot{A}_{+} \in L^{3 / 2}$ and let $\nabla \cdot A_{+}=\nabla \cdot \dot{A}_{+}=0$. 
Then there exists $T, 1 \leq T<\infty$ and there exists a unique solution $(u, A)$ of the system (1.3) such that $\left(u-u_{a}, A-A_{a}\right) \in X([T, \infty))$. Furthermore $\nabla\left(A-A_{a}\right), \partial_{t}\left(A-A_{a}\right) \in \mathcal{C}\left([T, \infty), L^{2}\right)$ and $A$ satisfies the estimate

$$
\left\|\nabla\left(A-A_{a}\right)(t)\right\|_{2} \vee\left\|\partial_{t}\left(A-A_{a}\right)(t)\right\|_{2} \leq C t^{-3 / 2}(2+\ell n t)^{2}
$$

for some constant $C$ depending on $\left(u_{+}, A_{+}, \dot{A}_{+}\right)$and for all $t \geq T$.

Remark 1.1. The only smallness conditions bear on $\left\|x w_{+}\right\|_{4}$ and on $\left\|w_{+}\right\|_{3}$ and are required by the magnetic interaction and the Hartree interaction (1.2) respectively. In particular there is no smallness condition on $\left(A_{+}, \dot{A}_{+}\right)$.

Remark 1.2. The assumptions $A_{+}, x \cdot A_{+} \in L^{3}$ and $\dot{A}_{+}, x \cdot \dot{A}_{+} \in L^{3 / 2}$ serve to exclude the occurrence of constant terms in $A_{+}, x \cdot A_{+}, \dot{A}_{+}, x \cdot \dot{A}_{+}$ and of terms linear in $x$ in $A_{+}, x \cdot A_{+}$, but are otherwise implied by the $W_{1}^{1}$ assumptions on those quantities through Sobolev inequalities.

Remark 1.3. The assumptions on $A_{+}, \dot{A}_{+}$imply that $\omega^{1 / 2} A_{+}, \omega^{-1 / 2} \dot{A}_{+}$ $\in H^{1}$ through Sobolev inequalities. As a consequence the free wave solution $A_{0}$ defined by (1.11) belongs to $L^{4}\left(\mathbb{R}, W_{4}^{1}\right)$ by Strichartz inequalities, with $\partial_{t} A_{0} \in L^{4}\left(\mathbb{R}, L^{4}\right)[3]$. In particular $A_{0}$ satisfies the local in time regularity of $B$ required in the definition of the space $X(\cdot)$. Furthermore $\nabla A_{+}, \dot{A}_{+} \in L^{2}$ and therefore $\nabla A_{0}, \partial_{t} A_{0} \in\left(\mathcal{C} \cap L^{\infty}\right)\left(\mathbb{R}, L^{2}\right)$, namely $A_{0}$ is a finite energy solution of the wave equation.

\section{$\S 2 . \quad$ The Cauchy Problem at Infinite Initial Time}

In this section we perform the first step of the construction of solutions of the system (1.3) as described in the introduction, namely we construct solutions $(v, B)$ of the system (1.4) defined in a neighbourhood of infinity in time and tending to zero at infinity under suitable regularity and decay assumptions on the asymptotic functions $\left(u_{a}, A_{a}\right)$ and on the remainders $R_{i}$. As a preliminary to that study, we need to solve the Cauchy problem with finite initial time for the linearized system (1.7). That system consists of two independent equations. The second one is simply a wave equation with an inhomogeneous term and the Cauchy problem with finite or infinite initial time for it is readily solved under suitable assumptions on the inhomogeneous term, which will be fulfilled in the applications. The first one is a Schrödinger equation with time dependent 
magnetic and scalar potentials and with time dependent inhomogeneity, which we rewrite in a more concise form and with slightly different notation as

$$
i \partial_{t} v=-(1 / 2) \Delta_{A} v+V v+f
$$

We first give some preliminary results on the Cauchy problem with finite initial time for that equation at the level of regularity of $H^{2}$. The following proposition is a minor variation of Proposition 3.2 in [7].

Proposition 2.1. Let $I$ be an interval, let $A \in \mathcal{C}\left(I, L^{4}+L^{\infty}\right), \partial_{t} A \in$ $L_{\text {loc }}^{1}\left(I, L^{4}+L^{\infty}\right), V \in \mathcal{C}\left(I, L^{2}+L^{\infty}\right), \partial_{t} V \in L_{l o c}^{1}\left(I, L^{2}+L^{\infty}\right), f \in \mathcal{C}\left(I, L^{2}\right)$ and $\partial_{t} f \in L_{l o c}^{1}\left(I, L^{2}\right)$. Let $t_{0} \in I$ and $v_{0} \in H^{2}$. Then

(1) There exists a unique solution $v \in \mathcal{C}\left(I, H^{2}\right) \cap \mathcal{C}^{1}\left(I, L^{2}\right)$ of $(2.1)$ in I with $v\left(t_{0}\right)=v_{0}$. That solution is actually unique in $\mathcal{C}\left(I, H^{1}\right)$. For all $t \in I$, the following equality holds:

$$
\|v(t)\|_{2}^{2}-\left\|v_{0}\right\|_{2}^{2}=\int_{t_{0}}^{t} d t^{\prime} 2 \operatorname{Im}<v, f>\left(t^{\prime}\right) .
$$

(2) Let in addition $A \in L_{l o c}^{2}\left(I, L^{\infty}\right), \nabla A \in L_{l o c}^{1}\left(I, L^{\infty}\right)$ and $V \in L_{l o c}^{1}\left(I, L^{\infty}\right)$. Then for all $t \in I$, the following equality holds:

$$
\left\|\partial_{t} v(t)\right\|_{2}^{2}-\left\|\left(-(1 / 2) \Delta_{A} v_{0}+V v_{0}+f\right)\left(t_{0}\right)\right\|_{2}^{2}=\int_{t_{0}}^{t} d t^{\prime} 2 \operatorname{Im}<\partial_{t} v, f_{1}>\left(t^{\prime}\right)
$$

where

$$
f_{1}=i\left(\partial_{t} A\right) \cdot \nabla_{A} v+\left(\partial_{t} V\right) v+\partial_{t} f
$$

Furthermore the solution is unique in $\mathcal{C}\left(I, L^{2}\right)$.

We shall make an essential use of the well-known Strichartz inequalities for the Schrödinger equation [2], [10], [22], which we recall for completeness. We define

$$
U(t)=\exp (i(t / 2) \Delta)
$$

A pair of Hölder exponents $(q, r)$ will be called admissible if $0 \leq 2 / q=3 / 2-$ $3 / r \leq 1$. For any $r, 1 \leq r \leq \infty$, we define $\bar{r}$ by $1 / r+1 / \bar{r}=1$. 
Lemma 2.1. The following inequalities hold.

(1) For any admissible pair $(q, r)$ and for any $u \in L^{2}$

$$
\left\|U(t) u ; L^{q}\left(\mathbb{R}, L^{r}\right)\right\| \leq C\|u\|_{2} .
$$

(2) Let $I$ be an interval and let $t_{0} \in I$. Then for any admissible pairs $\left(q_{i}, r_{i}\right)$, $i=1,2$,

$$
\left\|\int_{t_{0}}^{t} d t^{\prime} U\left(\cdot-t^{\prime}\right) f\left(t^{\prime}\right) ; L^{q_{1}}\left(I, L^{r_{1}}\right)\right\| \leq C\left\|f ; L^{\bar{q}_{2}}\left(I, L^{\bar{r}_{2}}\right)\right\| .
$$

In addition to the Strichartz inequalities for the Schrödinger equation, we shall need special cases of the Strichartz inequalities for the wave equation [3], [10]. Let $I$ be an interval, let $t_{0} \in I$ and let $B\left(t_{0}\right)=\partial_{t} B\left(t_{0}\right)=0$. Then

(2.8) $\left\|B ; L^{4}\left(I, L^{4}\right)\right\| \leq C\left\|\square B ; L^{4 / 3}\left(I, L^{4 / 3}\right)\right\|$,

(2.9) $\left\|\nabla B ; L^{4}\left(I, L^{4}\right)\right\| \vee\left\|\partial_{t} B ; L^{4}\left(I, L^{4}\right)\right\| \leq C\left\|\nabla \square B ; L^{4 / 3}\left(I, L^{4 / 3}\right)\right\|$,

(2.10) $\operatorname{Sup}_{t \in I}\left(\|\nabla B(t)\|_{2} \vee\left\|\partial_{t} B(t)\right\|_{2}\right) \leq\left\|\square B ; L^{1}\left(I, L^{2}\right)\right\|$.

We now begin the construction of solutions of the system (1.4). For any $T, t_{0}$ with $1 \leq T<t_{0} \leq \infty$, we denote by $I$ the interval $I=\left[T, t_{0}\right]$ and for any $t \in I$, we denote by $J$ the interval $J=\left[t, t_{0}\right]$. In all this section, we denote by $h$ a function in $\mathcal{C}\left([1, \infty), \mathbb{R}^{+}\right)$such that for some $\lambda>0$, the function $\bar{h}(t) \equiv t^{\lambda} h(t)$ is non increasing and tends to zero as $t \rightarrow \infty$, and we denote by $j, k$ nonnegative integers.

We shall make repeated use of the following lemma.

Lemma 2.2. Let $1 \leq q, q_{k} \leq \infty(1 \leq k \leq n)$ be such that

$$
\mu \equiv 1 / q-\sum_{k} 1 / q_{k} \geq 0 .
$$

Let $f_{k} \in L^{q_{k}}(I)$ satisfy

$$
\left\|f_{k} ; L^{q_{k}}(J)\right\| \leq N_{k} h(t)
$$

for $1 \leq k \leq n$, for some constants $N_{k}$ and for all $t \in I$.

Let $\rho \geq 0$ such that $n \lambda+\rho>\mu$. Then the following inequality holds for all $t \in I$

$$
\left\|\left(\prod_{k} f_{k}\right) t^{-\rho} ; L^{q}(J)\right\| \leq C\left(\prod_{k} N_{k}\right) h(t)^{n} t^{\mu-\rho}
$$


where

$$
C=\left(1-2^{-q(n \lambda+\rho-\mu)}\right)^{-1 / q}
$$

Proof. For $t \in I$, we define $I_{j}=\left[t 2^{j}, t 2^{j+1}\right] \cap I$ so that $J=\cup_{j \geq 0} I_{j}$. We then rewrite $L^{q}(J)=\ell_{j}^{q}\left(L^{q}\left(I_{j}\right)\right)$. We estimate

$$
\begin{aligned}
\left\|\left(\prod_{k} f_{k}\right) t^{-\rho} ; L^{q}(J)\right\| & \leq\left\|\left(\prod_{k}\left\|f_{k} ; L^{q_{k}}\left(I_{j}\right)\right\|\right)\right\| t^{-\rho} ; L^{1 / \mu}\left(I_{j}\right)\left\|; \ell_{j}^{q}\right\| \\
& \leq\left(\prod_{k} N_{k}\right)\left\|h\left(t 2^{j}\right)^{n}\left(t 2^{j}\right)^{-\rho+\mu} ; \ell_{j}^{q}\right\| \\
& \leq\left(\prod_{k} N_{k}\right) \bar{h}(t)^{n} t^{-n \lambda-\rho+\mu}\left\|2^{j(-n \lambda-\rho+\mu)} ; \ell_{j}^{q}\right\|
\end{aligned}
$$

from which (2.12) follows.

Remark 2.1. In some special cases, the dyadic decomposition is not needed for the proof of Lemma 2.2. For instance if all the $q_{k}$ are infinite, one can estimate

$$
\begin{aligned}
& \left\|h(t)^{n} t^{-\rho}\right\|_{q} \leq \bar{h}(t)^{n}\left\|t^{-\rho-n \lambda}\right\|_{q} \\
& \leq C \bar{h}(t)^{n} t^{-\rho-n \lambda+1 / q}=C h(t)^{n} t^{-\rho+\mu}
\end{aligned}
$$

by a direct application of Hölder's inequality in $J$. The same situation occurs if $\rho>\mu$.

In order to estimate the Hartree interaction term (1.2), we shall use the following Lemma. We recall that $\delta(r)=3 / 2-3 / r$.

Lemma 2.3. The following estimates hold.

$$
\left\|g\left(\bar{v}_{1} v_{2}\right) v_{3}\right\|_{\bar{r}_{4}} \leq C \prod_{1 \leq i \leq 3}\left\|v_{i}\right\|_{r_{i}}
$$

for $0 \leq \delta_{i}=\delta\left(r_{i}\right) \leq 1,1 \leq i \leq 4, \sum \delta_{i}=1,0<\delta_{1}+\delta_{2}<1$.

$$
\left\|g\left(\bar{v}_{1} v_{2}\right)\right\|_{\infty} \leq C\left\|v_{2}\right\|_{r_{2}}\left(\left\|v_{1}\right\|_{r_{1+}}\left\|v_{1}\right\|_{r_{1-}}\right)^{1 / 2}
$$

for $0<3 / r_{1}=2-3 / r_{2} \leq 2,1 / r_{1 \pm}=(1 \mp \varepsilon) / r_{1}, \varepsilon>0$. 
Proof. Part (1) follows from the Hölder and Hardy-Littlewood-Sobolev inequalities.

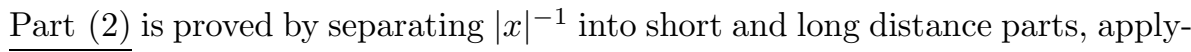
ing the Hölder inequality, and optimizing the result with respect to the point of separation (see [1]).

We can now state the main result of this section.

Proposition 2.2. $\quad$ Let $h$ be defined as above with $\lambda=3 / 8$ and let $X(\cdot)$ be defined by (1.17). Let $u_{a}, A_{a}, R_{1}$ and $R_{2}$ be sufficiently regular (for the following estimates to make sense) and satisfy the estimates

$$
\left\|\partial_{t}^{j} \nabla^{k} u_{a}(t)\right\|_{r} \leq c t^{-\delta(r)} \quad \text { for } 2 \leq r \leq \infty
$$

and in particular

$$
\begin{aligned}
& \left\|u_{a}\right\|_{3} \leq c_{3} t^{-1 / 2}, \quad\left\|\nabla u_{a}\right\|_{4} \leq c_{4} t^{-3 / 4}, \\
& \left\|\nabla^{2} u_{a}(t)\right\|_{4} \vee\left\|\partial_{t} \nabla u_{a}(t)\right\|_{4} \leq c t^{-3 / 4}, \\
& \left\|\partial_{t}^{j} \nabla^{k} A_{a}(t)\right\|_{\infty} \leq a t^{-1} \\
& \left\|\partial_{t}^{j} \nabla^{k} R_{1} ; L^{1}\left([t, \infty), L^{2}\right)\right\| \leq r_{1} h(t), \\
& \left\|R_{2} ; L^{4 / 3}\left([t, \infty), W_{4 / 3}^{1}\right)\right\| \leq r_{2} h(t),
\end{aligned}
$$

for $0 \leq j+k \leq 1$, for some constants $c, c_{3}, c_{4}, a, r_{1}$ and $r_{2}$ with $c_{3}$ and $c_{4}$ sufficiently small and for all $t \geq T_{0} \geq 1$. Then there exists $T, T_{0} \leq T<\infty$ and there exists a unique solution $(v, B)$ of the system $(1.4)$ in $X([T, \infty))$. If in addition

$$
\left\|R_{2} ; L^{1}\left([t, \infty), L^{2}\right)\right\| \leq r_{2} t^{-1 / 2} h(t)
$$

for all $t \geq T$, then $\nabla B, \partial_{t} B \in \mathcal{C}\left([T, \infty), L^{2}\right)$ and $B$ satisfies the estimate

$$
\|\nabla B(t)\|_{2} \vee\left\|\partial_{t} B(t)\right\|_{2} \leq C\left(t^{-1 / 2}+t^{1 / 4} h(t)\right) h(t)
$$

for some constant $C$ and for all $t \geq T$.

Proof. We follow the sketch given in the introduction. Let $T_{0} \leq T<\infty$ and let $(v, B) \in X([T, \infty))$. In particular $(v, B)$ satisfies

$$
\begin{aligned}
& \|v(t)\|_{2} \leq N_{0} h(t) \\
& \left\|v ; L^{4}\left(J, L^{3}\right)\right\| \vee\left\|v ; L^{8 / 3}\left(J, L^{4}\right)\right\| \leq N_{1} h(t)
\end{aligned}
$$




$$
\begin{aligned}
& \left\|B ; L^{4}\left(J, L^{4}\right)\right\| \leq N_{2} h(t) \\
& \left\|\partial_{t} v(t)\right\|_{2} \leq N_{3} h(t) \\
& \left\|\nabla v ; L^{4}\left(J, L^{3}\right)\right\| \vee\left\|\nabla v ; L^{8 / 3}\left(J, L^{4}\right)\right\| \leq N_{4} h(t) \\
& \|\Delta v(t)\|_{2} \leq N_{5} h(t) \\
& \left\|\nabla B ; L^{4}\left(J, L^{4}\right)\right\| \vee\left\|\partial_{t} B ; L^{4}\left(J, L^{4}\right)\right\| \leq N_{6} h(t)
\end{aligned}
$$

for some constants $N_{i}, 0 \leq i \leq 6$ and for all $t \geq T$, with $J=[t, \infty)$. Furthermore from (2.25) (2.30) it follows that

$$
\|\nabla v(t)\|_{2} \leq\left(N_{0} N_{5}\right)^{1 / 2} h(t) \equiv N_{1 / 2} h(t)
$$

for all $t \geq T_{0}$. We first construct a solution $\left(v^{\prime}, B^{\prime}\right)$ of the system (1.7) in $X([T, \infty))$. For that purpose, we take $t_{0}, T<t_{0}<\infty$ and we solve the system $(1.7)$ in $X(I)$ where $I=\left[T, t_{0}\right]$ with initial condition $\left(v^{\prime}, B^{\prime}\right)\left(t_{0}\right)=0$. Let $\left(v_{t_{0}}^{\prime}, B_{t_{0}}^{\prime}\right)$ be the solution thereby obtained. The existence of $v_{t_{0}}^{\prime}$ follows from Proposition 2.1 with $V=g\left(|u|^{2}\right)$ and $f=G_{1}-R_{1}$. We want to take the limit of $\left(v_{t_{0}}^{\prime}, B_{t_{0}}^{\prime}\right)$ as $t_{0} \rightarrow \infty$ and for that purpose we need estimates of $\left(v_{t_{0}}^{\prime}, B_{t_{0}}^{\prime}\right)$ in $X(I)$ that are uniform in $t_{0}$. Omitting the subscript $t_{0}$ for brevity we define

$$
\begin{aligned}
& N_{0}^{\prime}=\operatorname{Sup}_{t \in I} h(t)^{-1}\left\|v^{\prime}(t)\right\|_{2} \\
& N_{1}^{\prime}=\operatorname{Sup}_{t \in I} h(t)^{-1}\left(\left\|v^{\prime} ; L^{4}\left(J, L^{3}\right)\right\| \vee\left\|v^{\prime} ; L^{8 / 3}\left(J, L^{4}\right)\right\|\right) \\
& N_{2}^{\prime}=\operatorname{Sup}_{t \in I} h(t)^{-1}\left\|B^{\prime} ; L^{4}\left(J, L^{4}\right)\right\| \\
& N_{3}^{\prime}=\operatorname{Sup}_{t \in I} h(t)^{-1}\left\|\partial_{t} v^{\prime}(t)\right\|_{2} \\
& N_{4}^{\prime}=\operatorname{Sup}_{t \in I} h(t)^{-1}\left(\left\|\nabla v^{\prime} ; L^{4}\left(J, L^{3}\right)\right\| \vee\left\|\nabla v^{\prime} ; L^{8 / 3}\left(J, L^{4}\right)\right\|\right) \\
& N_{5}^{\prime}=\operatorname{Sup}_{t \in I} h(t)^{-1}\left\|\Delta v^{\prime}(t)\right\|_{2} \\
& N_{6}^{\prime}=\operatorname{Sup}_{t \in I} h(t)^{-1}\left(\left\|\nabla B^{\prime} ; L^{4}\left(J, L^{4}\right)\right\| \vee\left\|\partial_{t} B^{\prime} ; L^{4}\left(J, L^{4}\right)\right\|\right)
\end{aligned}
$$

where $J=[t, \infty) \cap I$ and we set out to estimate the various $N_{i}^{\prime}$. We also define the auxiliary quantities

$$
\begin{aligned}
& N_{1 / 2}^{\prime}=\operatorname{Sup}_{t \in I} h(t)^{-1}\left\|\nabla v^{\prime}(t)\right\|_{2} \\
& \tilde{N}_{1 / 2}^{\prime}=\operatorname{Sup}_{t \in I} h(t)^{-1}\left\|\nabla_{A} v^{\prime}(t)\right\|_{2}
\end{aligned}
$$

so that in particular $N_{1 / 2}^{\prime} \leq\left(N_{0}^{\prime} N_{5}^{\prime}\right)^{1 / 2}$. 
We shall use the notation

$$
\left\|f ; L^{q}\left(J, L^{r}\right)\right\|=\|\| f\left\|_{r} ; L^{q}(J)\right\|=\|\| f\left\|_{r}\right\|_{q},
$$

namely with the inner norm taken in $L^{r}\left(\mathbb{R}^{3}\right)$ and the outer norm taken in $L^{q}(J)$. Furthermore we shall use a shorthand notation for two important cases, namely

$$
\left\|\cdot ; L^{1}\left(J, L^{2}\right)\right\|=\|\cdot\|_{+} \quad \text { and } \quad\left\|\cdot ; L^{4 / 3}\left(J, L^{4 / 3}\right)\right\|=\|\cdot\|_{*} .
$$

We first estimate $N_{0}^{\prime}$, defined by (2.33). From (2.2) we obtain

$$
\left\|v^{\prime}(t)\right\|_{2} \leq\left\|G_{1}\right\|_{+}+\left\|R_{1}\right\|_{+}
$$

with $G_{1}$ defined by (1.5). We estimate

$$
\left\|B \cdot \nabla u_{a}\right\|_{+} \leq\|\| B\left\|_{4}\right\| \nabla u_{a}\left\|_{4}\right\|_{1} \leq C c_{4} N_{2} h(t)
$$

by Lemma 2.2,

$$
\begin{aligned}
\left\|B \cdot A_{a} u_{a}\right\|_{+} & \leq\|\| B\left\|_{4}\right\| A_{a}\left\|_{\infty}\right\| u_{a}\left\|_{4}\right\|_{1} \\
& \leq c a N_{2} h\left\|t^{-7 / 4}\right\|_{4 / 3} \leq c a N_{2} t^{-1} h(t), \\
\left\|B^{2} u_{a}\right\|_{+} & \leq\|\| B\left\|_{4}^{2}\right\| u_{a}\left\|_{\infty}\right\|_{1} \leq c N_{2}^{2} h^{2}\left\|t^{-3 / 2}\right\|_{2} \leq c N_{2}^{2} t^{-1} h(t)^{2}, \\
\left\|g\left(\bar{u}_{a} v\right) u_{a}\right\|_{+} & \leq C\|\| v\left\|_{2}\right\| u_{a}\left\|_{3}^{2}\right\|_{1} \leq C c_{3}^{2} N_{0} h(t)
\end{aligned}
$$

by Lemma 2.3, part (1) and Lemma 2.2,

$$
\begin{aligned}
\left\|g\left(|v|^{2}\right) u_{a}\right\|_{+} & \leq C\|\| v\left\|_{3}\right\| v\left\|_{2}\right\| u_{a}\left\|_{3}\right\|_{1} \\
& \leq C c_{3} N_{0} N_{1} t^{1 / 4} h(t)^{2}
\end{aligned}
$$

by Lemma 2.3, part (1) and Lemma 2.2 again.

Collecting the previous estimates yields

$$
\begin{aligned}
N_{0}^{\prime} \leq & C_{0}\left(c_{4} N_{2}+c a N_{2} T^{-1}+c_{3}^{2} N_{0}+c N_{2}^{2} T^{-1} h(T)\right. \\
& \left.+c N_{0} N_{1} T^{-1 / 8} \bar{h}(T)+r_{1}\right)
\end{aligned}
$$

which is of the form

$$
N_{0}^{\prime} \leq C_{0}\left(c_{4} N_{2}+c_{3}^{2} N_{0}+r_{1}+\left(o(1) ; N_{0}, N_{1}, N_{2}\right)\right)
$$

where $(o(1) ; \cdot, \cdots, \cdot)$ denotes a quantity depending on the variables indicated and tending to zero as $T \rightarrow \infty$ when those variables are fixed. 
We next estimate the Strichartz norms of $v^{\prime}$, namely $N_{1}^{\prime}$ defined by (2.34). By Lemma 2.1, in addition to the contribution of $G_{1}-R_{1}$ estimated above, we need to estimate

$$
i A \cdot \nabla v^{\prime}+(1 / 2) A^{2} v^{\prime}+g\left(|u|^{2}\right) v^{\prime}
$$

in some $L^{\bar{q}}\left(J, L^{\bar{r}}\right)$ for admissible $(q, r)$. We estimate

$$
\begin{aligned}
\left\|A_{a} \cdot \nabla v^{\prime}\right\|_{+} & \leq a N_{1 / 2}^{\prime}\left\|t^{-1} h\right\|_{1} \leq 3 a N_{1 / 2}^{\prime} h(t), \\
\left\|A_{a}^{2} v^{\prime}\right\|_{+} & \leq a^{2} N_{0}^{\prime}\left\|t^{-2} h\right\|_{1} \leq a^{2} N_{0}^{\prime} t^{-1} h(t), \\
\left\|B \cdot \nabla v^{\prime} ; L^{8 / 5}\left(J, L^{4 / 3}\right)\right\| & \leq\|\| B\left\|_{4}\right\| \nabla v^{\prime}\left\|_{2}\right\|_{8 / 5} \leq C N_{2} N_{1 / 2}^{\prime} h(t) \bar{h}(t),
\end{aligned}
$$

by Lemma 2.2 ,

$$
\left\|B^{2} v^{\prime} ; L^{2}\left(J, L^{6 / 5}\right)\right\| \leq C\|\| B\left\|_{4}^{3 / 2}\right\| \nabla B\left\|_{4}^{1 / 2}\right\| v^{\prime}\left\|_{2}\right\|_{2} \leq C N_{2}^{3 / 2} N_{6}^{1 / 2} N_{0}^{\prime} h(t)^{3}
$$

by Sobolev inequalities and Lemma 2.2 ,

$$
\left\|g\left(\left|u_{a}\right|^{2}\right) v^{\prime}\right\|_{+} \leq\|\| g\left(\left|u_{a}\right|^{2}\right)\left\|_{\infty}\right\| v^{\prime}\left\|_{2}\right\|_{1} \leq C c^{2} N_{0}^{\prime} h(t)
$$

by Lemma 2.3, part (2),

$$
\left\|g\left(|v|^{2}\right) v^{\prime} ; L^{4 / 3}\left(J, L^{3 / 2}\right)\right\| \leq C\|\| v\left\|_{3}\right\| v\left\|_{2}\right\| v^{\prime}\left\|_{2}\right\|_{4 / 3} \leq C N_{0} N_{1} N_{0}^{\prime} t^{1 / 2} h(t)^{3}
$$

by Lemma 2.3, part (1) and Lemma 2.2. The term $g\left(\bar{u}_{a} v\right) v^{\prime}$ need not be considered because it is controlled by the previous ones.

Collecting the previous estimates yields

$$
\begin{aligned}
N_{1}^{\prime} \leq & C_{1}\left\{c_{4} N_{2}+c a N_{2} T^{-1}+c_{3}^{2} N_{0}+c N_{2}^{2} T^{-1} h(T)+c N_{0} N_{1} T^{-1 / 8} \bar{h}(T)\right. \\
& +\left(c^{2}+a^{2} T^{-1}+N_{2}^{3 / 2} N_{6}^{1 / 2} h(T)^{2}+N_{0} N_{1} T^{-1 / 4} \bar{h}(T)^{2}\right) N_{0}^{\prime}+r_{1} \\
& \left.+a N_{1 / 2}^{\prime}+N_{2} N_{1 / 2}^{\prime} \bar{h}(T)\right\}
\end{aligned}
$$

which is of the form

$$
\begin{aligned}
N_{1}^{\prime} \leq C_{1}( & c_{4} N_{2}+c_{3}^{2} N_{0}+c^{2} N_{0}^{\prime}+a N_{1 / 2}^{\prime}+r_{1} \\
& \left.+\left(o(1) ; N_{0}, N_{1}, N_{2}, N_{6}, N_{0}^{\prime}, N_{1 / 2}^{\prime}\right)\right)
\end{aligned}
$$

We now turn to the estimates of $B^{\prime}$. We first estimate $B^{\prime}$ in $L^{4}\left(J, L^{4}\right)$, namely we estimate $N_{2}^{\prime}$ defined by (2.35), by the use of (1.5) (2.8). For that purpose 
we estimate $G_{2}$ in $L^{4 / 3}\left(J, L^{4 / 3}\right)$. The linear terms in $v$ are estimated by

$$
\begin{aligned}
\left\|\bar{v} \nabla u_{a}\right\|_{*} & \leq\|\| v\left\|_{2}\right\| \nabla u_{a}\left\|_{4}\right\|_{4 / 3} \\
& \leq c_{4} N_{0}\left\|t^{-3 / 4} h\right\|_{4 / 3} \leq 2 c_{4} N_{0} h(t), \\
\left\|\bar{v} A_{a} u_{a}\right\|_{*} & \leq\|\| v\left\|_{2}\right\| A_{a}\left\|_{\infty}\right\| u_{a}\left\|_{4}\right\|_{4 / 3} \\
& \leq a c N_{0}\left\|t^{-7 / 4} h\right\|_{4 / 3} \leq a c N_{0} t^{-1} h(t) .
\end{aligned}
$$

The linear term in $B$ is estimated by

$$
\begin{aligned}
\left\|B\left|u_{a}\right|^{2}\right\|_{*} & \leq\|\| B\left\|_{4}\right\| u_{a}\left\|_{4}^{2}\right\|_{4 / 3} \\
& \leq c^{2} N_{2} h\left\|t^{-3 / 2}\right\|_{2} \leq c^{2} N_{2} t^{-1} h(t) .
\end{aligned}
$$

The quadratic terms in $v^{2}$ are estimated by

$$
\|\bar{v} \nabla v\|_{*} \leq\|\| v\left\|_{4}\right\| \nabla v\left\|_{2}\right\|_{4 / 3} \leq C N_{1} N_{1 / 2} h(t) \bar{h}(t),
$$

by Lemma 2.2,

$$
\begin{aligned}
\left\|A_{a}|v|^{2}\right\|_{*} & \leq\|\| v\left\|_{4}\right\| v\left\|_{2}\right\| A_{a}\left\|_{\infty}\right\|_{4 / 3} \\
& \leq a N_{0} N_{1} h\left\|t^{-1} h\right\|_{8 / 3} \leq a N_{0} N_{1} t^{-5 / 8} h(t)^{2} .
\end{aligned}
$$

The quadratic terms in $B v$ need not be considered because

$$
2\left|\bar{v} B u_{a}\right| \leq\left.|B| u_{a}\right|^{2}|+| B|v|^{2} \mid .
$$

The cubic term $B|v|^{2}$ is estimated by

$$
\begin{aligned}
\left\|B|v|^{2}\right\|_{*} & \leq\left\|B ; L^{4}\left(L^{4}\right)\right\|\left\|v ; L^{3}\left(L^{r}\right)\right\|^{3 / 2}\left\|v ; L^{\infty}\left(L^{6}\right)\right\|^{1 / 2} \\
& \leq C N_{2} N_{1}^{3 / 2} N_{1 / 2}^{1 / 2} h(t)^{3}
\end{aligned}
$$

where $3<r=18 / 5<4$ so that $(3, r)$ is an admissible pair and that the middle norm is controlled by $N_{1}$.

Collecting the previous estimates yields

$$
\begin{aligned}
N_{2}^{\prime} \leq C_{2} & \left\{c_{4} N_{0}+a c N_{0} T^{-1}+c^{2} N_{2} T^{-1}+r_{2}\right. \\
& \left.+N_{1} N_{1 / 2} \bar{h}(T)+a N_{0} N_{1} T^{-5 / 8} h(T)+N_{2} N_{1}^{3 / 2} N_{1 / 2}^{1 / 2} h(T)^{2}\right\}
\end{aligned}
$$

which is of the form

$$
N_{2}^{\prime} \leq C_{2}\left(c_{4} N_{0}+r_{2}+\left(o(1) ; N_{0}, N_{1}, N_{1 / 2}, N_{2}\right)\right)
$$


We next complete the estimates of $B^{\prime}$ by estimating $\nabla B^{\prime}$ and $\partial_{t} B^{\prime}$ in $L^{4}\left(J, L^{4}\right)$, namely we estimate $N_{6}^{\prime}$ defined by (2.39), through the use of (1.5) (2.9). For that purpose we estimate $\nabla G_{2}$ in $L^{4 / 3}\left(J, L^{4 / 3}\right)$. Now

$$
\begin{aligned}
\nabla G_{2}= & 2 P \operatorname{Im}\left((\nabla \bar{v}) \nabla_{A} v+(\nabla \bar{v}) \nabla_{A} u_{a}+\left(\nabla \bar{u}_{a}\right) \nabla_{A} v\right) \\
& -P(\nabla A)\left(|v|^{2}+2 \operatorname{Re} \bar{u}_{a} v\right)-P(\nabla B)\left|u_{a}\right|^{2}-2 P B \operatorname{Re} \bar{u}_{a} \nabla u_{a} .
\end{aligned}
$$

The estimate of $\nabla G_{2}$ in $L^{4 / 3}\left(J, L^{4 / 3}\right)$ proceeds exactly as that of $G_{2}$ in the same space, with one additional gradient acting on each factor in each term, except for two facts. First because of the symmetry of the quadratic form $P \operatorname{Im}\left(\bar{v}_{1} \nabla_{A} v_{2}\right)$, we can always ensure that no terms occur with two derivatives on $v$ or $u_{a}$. Second, the quadratic terms coming from $\bar{v} B u_{a}$ have to be estimated explicitly because they are no longer estimated by polarization. When hitting $v$, and additional gradient produces a replacement of $N_{0}$ by $N_{1 / 2}$ and of $N_{1}$ by $N_{4}$ in the estimates. When hitting $B$, it produces a replacement of $N_{2}$ by $N_{6}$. When hitting $u_{a}$ or $A_{a}$, it only requires higher regularity of these functions, but does not change the form of the estimates. With those remarks available, only the terms from $\nabla\left(\bar{v} B u_{a}\right)$ and from $B \nabla|v|^{2}$ need new estimates.

The linear terms in $v$ are estimated by

$$
\begin{aligned}
\left\|(\nabla \bar{v}) \nabla u_{a}\right\|_{*} & \leq 2 c_{4} N_{1 / 2} h(t), \\
\left\|(\nabla \bar{v}) A_{a} u_{a}\right\|_{*} & \leq a c N_{1 / 2} t^{-1} h(t), \\
\left\|\bar{v}\left(\nabla A_{a}\right) u_{a}+\bar{v} A_{a} \nabla u_{a}\right\|_{*} & \leq 2 a c N_{0} t^{-1} h(t) .
\end{aligned}
$$

The linear terms in $B$ are estimated by

$$
\begin{aligned}
\left\|(\nabla B)\left|u_{a}\right|^{2}\right\|_{*} & \leq c^{2} N_{6} t^{-1} h(t), \\
\left\|B \bar{u}_{a} \nabla u_{a}\right\|_{*} & \leq c^{2} N_{2} t^{-1} h(t) .
\end{aligned}
$$

The quadratic terms in $v^{2}$ are estimated by

$$
\begin{aligned}
\left\||\nabla v|^{2}\right\|_{*} & \leq\|\| \nabla v\left\|_{4}\right\| \nabla v\left\|_{2}\right\|_{4 / 3} \leq C N_{4} N_{1 / 2} h(t) \bar{h}(t), \\
\left\|(\nabla \bar{v}) A_{a} v\right\|_{*} & \leq\|\| \nabla v\left\|_{2}\right\| A_{a}\left\|_{\infty}\right\| v\left\|_{4}\right\|_{4 / 3} \leq a N_{1 / 2} N_{1} t^{-5 / 8} h(t)^{2}, \\
\left\|\left(\nabla A_{a}\right)|v|^{2}\right\|_{*} & \leq a N_{0} N_{1} t^{-5 / 8} h(t)^{2} .
\end{aligned}
$$

The quadratic terms in $B v$ are estimated by

$$
\begin{aligned}
\left\|(\nabla \bar{v}) B u_{a}\right\|_{*} & \leq\|\| \nabla v\left\|_{2}\right\| B\left\|_{4}\right\| u_{a}\left\|_{\infty}\right\|_{4 / 3} \\
& \leq c N_{1 / 2} N_{2} h\left\|t^{-3 / 2} h\right\|_{2} \leq c N_{1 / 2} N_{2} t^{-1} h(t)^{2}
\end{aligned}
$$


and similarly

$$
\begin{aligned}
\left\|\bar{v}(\nabla B) u_{a}\right\|_{*} & \leq c N_{0} N_{6} t^{-1} h(t)^{2}, \\
\left\|\bar{v} B \nabla u_{a}\right\|_{*} & \leq c N_{0} N_{2} t^{-1} h(t)^{2} .
\end{aligned}
$$

The cubic terms from $B|v|^{2}$ are estimated by

$$
\begin{aligned}
\left\|(\nabla B)|v|^{2}\right\|_{*} & \leq C N_{6} N_{1}^{3 / 2} N_{1 / 2}^{1 / 2} h(t)^{3}, \\
\|B \bar{v} \nabla v\|_{*} & \leq C\left\|B ; L^{4}\left(L^{4}\right)\right\|\left\|v ; L^{4}\left(L^{3}\right)\right\|^{1 / 2}\left\|\nabla v ; L^{4}\left(L^{3}\right)\right\|^{3 / 2} \\
& \leq C N_{2} N_{1}^{1 / 2} N_{4}^{3 / 2} h(t)^{3} .
\end{aligned}
$$

Collecting the previous estimates yields

$$
\begin{aligned}
N_{6}^{\prime} \leq & C_{6}\left\{c_{4} N_{1 / 2}+a c\left(N_{1 / 2}+N_{0}\right) T^{-1}+c^{2}\left(N_{2}+N_{6}\right) T^{-1}+r_{2}+N_{1 / 2} N_{4} \bar{h}(T)\right. \\
& +a\left(N_{1 / 2}+N_{0}\right) N_{1} T^{-5 / 8} h(T)+c\left(N_{2} N_{1 / 2}+N_{2} N_{0}+N_{6} N_{0}\right) T^{-1} h(T) \\
& \left.+\left(N_{6} N_{1}^{3 / 2} N_{1 / 2}^{1 / 2}+N_{2} N_{1}^{1 / 2} N_{4}^{3 / 2}\right) h(T)^{2}\right\}
\end{aligned}
$$

which is of the form

$$
N_{6}^{\prime} \leq C_{6}\left(c_{4} N_{1 / 2}+r_{2}+\left(o(1) ; N_{0}, N_{1}, N_{1 / 2}, N_{4}, N_{2}, N_{6}\right)\right) .
$$

We now come back to the estimates of $v^{\prime}$ and we first estimate $\partial_{t} v^{\prime}$ in $L^{2}$, namely we estimate $N_{3}^{\prime}$ defined by (2.36) by using (2.3). Here however we encounter a technical difficulty due to the fact that $B$ a priori does not satisfy the assumption $\nabla B \in L_{l o c}^{1}\left(I, L^{\infty}\right)$ needed in Proposition 2.1, part (2) in order to derive (2.3). We circumvent that difficulty by first regularizing $B$, introducing the associated solution $v^{\prime}$ which then satisfies (2.3), deriving the $N_{3}^{\prime}$ estimate for the auxiliary solution, and removing the regularization by a limiting procedure, which preserves the estimate. Here in order not to burden the proof with technicalities, we provide only the derivation of the estimates from (2.3) and we refer to the proof of Proposition 3.2, part (1) in [7] for the technical details. From (2.3) (2.4) with $V=g\left(|u|^{2}\right)$ and $f=G_{1}-R_{1}$, we obtain

$$
\left\|\partial_{t} v^{\prime}\right\|_{2} \leq\left\|i\left(\partial_{t} A\right) \cdot \nabla_{A} v^{\prime}+\left(\partial_{t} g\left(|u|^{2}\right)\right) v^{\prime}+\partial_{t} G_{1}-\partial_{t} R_{1}\right\|_{+}+\left\|\left(G_{1}-R_{1}\right)\left(t_{0}\right)\right\|_{2}
$$

with $G_{1}$ defined in (1.5).

We first estimate the terms containing $v^{\prime}$, starting with $i\left(\partial_{t} A\right) \cdot \nabla_{A} v^{\prime}$.

$$
\begin{aligned}
\left\|\left(\partial_{t} A_{a}\right) \cdot \nabla_{A} v^{\prime}\right\|_{+} & \leq\|\| \partial_{t} A_{a}\left\|_{\infty}\right\| \nabla_{A} v^{\prime}\left\|_{2}\right\|_{1} \\
& \leq a \widetilde{N}_{1 / 2}^{\prime}\left\|t^{-1} h\right\|_{1} \leq 3 a \widetilde{N}_{1 / 2}^{\prime} h(t),
\end{aligned}
$$




$$
\begin{aligned}
\left\|\left(\partial_{t} B\right) \cdot \nabla v^{\prime}\right\|_{+} & \leq\|\| \partial_{t} B\left\|_{4}\right\| \nabla v^{\prime}\left\|_{4}\right\|_{1} \leq C N_{6} N_{4}^{\prime} h(t) \bar{h}(t), \\
\left\|\left(\partial_{t} B\right) \cdot A_{a} v^{\prime}\right\|_{+} & \leq\|\| \partial_{t} B\left\|_{4}\right\| A_{a}\left\|_{\infty}\right\| v^{\prime}\left\|_{4}\right\|_{1} \\
& \leq a N_{6} N_{1}^{\prime} h^{2}\left\|t^{-1}\right\|_{8 / 3} \leq a N_{6} N_{1}^{\prime} t^{-5 / 8} h(t)^{2}, \\
\left\|\left(\partial_{t} B\right) \cdot B v^{\prime}\right\|_{+} & \leq C\|\| \partial_{t} B\left\|_{4}\left(\|B\|_{4}\|\nabla B\|_{4}^{3}\right)^{1 / 4}\right\| v^{\prime}\left\|_{4}\right\|_{1} \\
& \leq C N_{6}^{7 / 4} N_{2}^{1 / 4} N_{1}^{\prime} t^{1 / 8} h(t)^{3}
\end{aligned}
$$

by Lemma 2.2 .

We next estimate the terms coming from $\left(\partial_{t} g\left(|u|^{2}\right)\right) v^{\prime}$.

$$
\begin{aligned}
\left\|g\left(\bar{u}_{a} \partial_{t} u_{a}\right) v^{\prime}\right\|_{+} & \leq\|\| g\left(\bar{u}_{a} \partial_{t} u_{a}\right)\left\|_{\infty}\right\| v^{\prime}\left\|_{2}\right\|_{1} \leq C c^{2} N_{0}^{\prime} h(t), \\
\left\|g\left(\bar{u}_{a} \partial_{t} v\right) v^{\prime}\right\|_{+} & \leq\|\| g\left(\bar{u}_{a} \partial_{t} v\right)\left\|_{\infty}\right\| v^{\prime}\left\|_{2}\right\|_{1} \leq C c N_{3} N_{0}^{\prime} h(t)^{2}, \\
\left\|g\left(\left(\partial_{t} \bar{u}_{a}\right) v\right) v^{\prime}\right\|_{+} & \leq C c N_{0} N_{0}^{\prime} h(t)^{2}
\end{aligned}
$$

by Lemma 2.3 , part (2) and Lemma 2.2 ,

$$
\left\|g\left(\bar{v} \partial_{t} v\right) v^{\prime}\right\|_{+} \leq\|\| v\left\|_{3}\right\| \partial_{t} v\left\|_{2}\right\| v^{\prime}\left\|_{3}\right\|_{1} \leq C N_{1} N_{3} N_{1}^{\prime} t^{1 / 2} h(t)^{3}
$$

by Lemma 2.3, part (1) and Lemma 2.2 .

We next estimate $\partial_{t} G_{1}$. The estimates are similar to those performed when estimating $v^{\prime}$ in $L^{2}$, with an additional time derivative acting on each factor in each term. This has the effect of requiring more regularity on $\left(A_{a}, u_{a}\right)$ when that derivative hits $\left(A_{a}, u_{a}\right)$, without changing the form the estimate, and of replacing one factor $N_{2}$ by $N_{6}$ when that derivative hits $B$ and one factor $N_{0}$ by $N_{3}$ when that derivative hits $v$. Thus we obtain

$$
\begin{aligned}
\left\|\left(\partial_{t} B\right) \cdot \nabla_{A_{a}} u_{a}\right\|_{+} & \leq N_{6}\left(C c_{4}+a c t^{-1}\right) h(t), \\
\left\|B \cdot \partial_{t} \nabla_{A_{a}} u_{a}\right\|_{+} & \leq c N_{2}\left(C+a t^{-1}\right) h(t), \\
\left\|\left(\partial_{t} B\right) B u_{a}\right\|_{+} & \leq c N_{2} N_{6} t^{-1} h(t)^{2}, \\
\left\|B^{2} \partial_{t} u_{a}\right\|_{+} & \leq c N_{2}^{2} t^{-1} h(t)^{2} . \\
\left\|\partial_{t}\left(g\left(\bar{u}_{a} v\right) u_{a}\right)\right\|_{+} & \leq C c_{3}\left(c_{3} N_{3}+c N_{0}\right) h(t) \\
\left\|\partial_{t}\left(g\left(|v|^{2}\right) u_{a}\right)\right\|_{+} & \leq C N_{1}\left(c_{3} N_{3}+c N_{0}\right) t^{1 / 4} h(t)^{2} .
\end{aligned}
$$

We finally estimate $\left\|\partial_{t} v^{\prime}\left(t_{0}\right)\right\|_{2}$ and for that purpose we need pointwise (in time) estimates of $R_{1}$ and of $B$. Now from (2.21) it follows that

$$
\left\|R_{1}(t)\right\|_{2} \leq\left\|\partial_{t} R_{1}\right\|_{+} \leq r_{1} h(t)
$$

while from $(2.27)(2.31)$

$$
\|B(t)\|_{4}^{4} \leq 4 \int_{t}^{\infty} d t^{\prime}\left\|B\left(t^{\prime}\right)\right\|_{4}^{3}\left\|\partial_{t} B\left(t^{\prime}\right)\right\|_{4} \leq 4 N_{2}^{3} N_{6} h(t)^{4}
$$


and therefore

$$
\|B(t)\|_{4} \leq \widetilde{N}_{2} h(t) \equiv \sqrt{2}\left(N_{2}^{3} N_{6}\right)^{1 / 4} h(t) .
$$

We then estimate

$$
\begin{gathered}
\left\|G_{1}\right\|_{2} \leq \| \\
\quad B\left\|_{4}\left(\left\|\nabla u_{a}\right\|_{4}+\left\|A_{a}\right\|_{\infty}\left\|u_{a}\right\|_{4}\right)+\right\| B\left\|_{4}^{2}\right\| u_{a} \|_{\infty} \\
\quad+\left\|g\left(|v|^{2}+2 \operatorname{Re} \bar{u}_{a} v\right)\right\|_{6}\left\|u_{a}\right\|_{3} \\
\leq c \widetilde{N}_{2}\left(1+a t^{-1}\right) t^{-3 / 4} h(t)+c \widetilde{N}_{2}^{2} t^{-3 / 2} h(t)^{2} \\
\quad+C\left(c_{3}^{2} N_{0} t^{-1} h(t)+c_{3} N_{0}^{3 / 2} N_{1 / 2}^{1 / 2} t^{-1 / 2} h(t)^{2}\right)
\end{gathered}
$$

by Lemma 2.3 for the terms containing $g$ and the definitions.

Collecting the previous estimates and in particular (2.52) (2.53) taken at $t_{0} \geq t$, we obtain

$$
\begin{aligned}
N_{3}^{\prime} \leq & C_{3}\left\{a \widetilde{N}_{1 / 2}^{\prime}+c_{4} N_{6}+c N_{2}+c a\left(N_{6}+N_{2}\right) T^{-1}+c_{3}^{2} N_{3}+c^{2}\left(N_{0}+N_{0}^{\prime}\right)+r_{1}\right. \\
& +N_{6} N_{4}^{\prime} \bar{h}(T)+a N_{6} N_{1}^{\prime} T^{-5 / 8} h(T)+c N_{2}\left(N_{6}+N_{2}\right) T^{-1} h(T) \\
& +c\left(N_{3}+N_{0}\right) N_{0}^{\prime} h(T)+c\left(N_{3}+N_{0}\right) N_{1} T^{-1 / 8} \bar{h}(T) \\
& +N_{6}^{7 / 4} N_{2}^{1 / 4} N_{1}^{\prime} T^{-1 / 4} h(T) \bar{h}(T)+N_{1} N_{3} N_{1}^{\prime} T^{-1 / 4} \bar{h}(T)^{2} \\
& +c \widetilde{N}_{2}\left(1+a T^{-1}\right) T^{-3 / 4}+c \widetilde{N}_{2}^{2} T^{-3 / 2} h(T) \\
& \left.+c_{3}^{2} N_{0} T^{-1}+c_{3} N_{0}^{3 / 2} N_{1 / 2}^{1 / 2} T^{-1 / 2} h(T)\right\}
\end{aligned}
$$

which is of the form

$$
\begin{aligned}
N_{3}^{\prime} \leq & C_{3}\left(a \widetilde{N}_{1 / 2}^{\prime}+c_{4} N_{6}+c N_{2}+c_{3}^{2} N_{3}+c^{2}\left(N_{0}+N_{0}^{\prime}\right)+r_{1}\right. \\
& \left.+\left(o(1) ; N_{0}, N_{0}^{\prime}, N_{1}, N_{1}^{\prime}, N_{1 / 2}, N_{2}, \widetilde{N_{2}}, N_{3}, N_{4}^{\prime}, N_{6}\right)\right) .
\end{aligned}
$$

We next estimate $\left\|\Delta_{A} v^{\prime}\right\|_{2}$. From (1.7), we obtain

$$
\begin{aligned}
\left\|\Delta_{A} v^{\prime}\right\| & \leq 2\left(\left\|\partial_{t} v^{\prime}\right\|_{2}+\left\|g\left(|u|^{2}\right) v^{\prime}\right\|_{2}+\left\|G_{1}\right\|_{2}+\left\|R_{1}\right\|_{2}\right) \\
& \leq 2\left(N_{3}^{\prime}+r_{1}\right) h(t)+2\left\|g\left(|u|^{2}\right) v^{\prime}\right\|_{2}+2\left\|G_{1}\right\|_{2} .
\end{aligned}
$$

Furthermore

$$
\begin{aligned}
& \left\|g\left(|u|^{2}\right) v^{\prime}\right\|_{2}+\left\|G_{1}\right\|_{2} \leq\left\|g\left(|u|^{2}\right)\right\|_{\infty}\left\|v^{\prime}\right\|_{2}+\left\|G_{1}\right\|_{2} \\
& \leq c \widetilde{N}_{2}\left(1+a t^{-1}\right) t^{-3 / 4} h(t)+c \widetilde{N}_{2}^{2} t^{-3 / 2} h(t)^{2} \\
& \quad+C\left(c^{2} N_{0}^{\prime} t^{-1} h(t)+N_{0} N_{1 / 2} N_{0}^{\prime} h(t)^{3}+c_{3}^{2} N_{0} t^{-1} h(t)\right. \\
& \left.\quad+c_{3} N_{0}^{3 / 2} N_{1 / 2}^{1 / 2} t^{-1 / 2} h(t)^{2}\right) \equiv M_{1} h(t)
\end{aligned}
$$


by Lemma 2.3 for the terms containing $g$ and by (2.53), so that

$$
\begin{aligned}
\left\|\Delta_{A} v^{\prime}(t)\right\|_{2} & \leq 2\left(N_{3}^{\prime}+r_{1}+M_{1}\right) h(t) \\
& =2\left(N_{3}^{\prime}+r_{1}+\left(o(1) ; \widetilde{N}_{2}, N_{0}, N_{0}^{\prime}, N_{1 / 2}\right)\right) h(t) .
\end{aligned}
$$

As a consequence,

$$
\begin{aligned}
\tilde{N}_{1 / 2}^{\prime} & \leq\left(2 N_{0}^{\prime}\left(N_{3}^{\prime}+r_{1}+M_{1}(T)\right)\right)^{1 / 2} \\
& \leq\left(2 N_{0}^{\prime}\left(N_{3}^{\prime}+r_{1}+\left(o(1) ; \tilde{N}_{2}, N_{0}, N_{0}^{\prime}, N_{1 / 2}\right)\right)\right)^{1 / 2} .
\end{aligned}
$$

We next estimate $\left\|\Delta v^{\prime}(t)\right\|_{2}$, namely $N_{5}^{\prime}$ defined by (2.38). From

$$
\Delta_{A} v^{\prime}=\Delta v^{\prime}-2 i A_{a} \cdot \nabla_{A} v^{\prime}-2 i B \cdot \nabla v^{\prime}+\left(A_{a}^{2}-B^{2}\right) v^{\prime}
$$

we obtain

$$
\begin{aligned}
\left\|\Delta v^{\prime}\right\|_{2} \leq & \left\|\Delta_{A} v^{\prime}\right\|_{2}+2\left\|A_{a}\right\|_{\infty}\left\|\nabla_{A} v^{\prime}\right\|_{2}+\left\|A_{a}\right\|_{\infty}^{2}\left\|v^{\prime}\right\|_{2} \\
& +2\|B\|_{4}\left\|\nabla v^{\prime}\right\|_{4}+\|B\|_{4}^{2}\left\|v^{\prime}\right\|_{\infty} .
\end{aligned}
$$

Now

$$
\begin{aligned}
\left\|\nabla v^{\prime}\right\|_{4} & \leq C\left\|\Delta v^{\prime}\right\|_{2}^{7 / 8}\left\|v^{\prime}\right\|_{2}^{1 / 8}, \\
\left\|v^{\prime}\right\|_{\infty} & \leq C\left\|\Delta v^{\prime}\right\|_{2}^{3 / 4}\left\|v^{\prime}\right\|_{2}^{1 / 4},
\end{aligned}
$$

and therefore

$$
\begin{aligned}
\left\|\Delta v^{\prime}\right\|_{2} \leq & (1+\varepsilon)\left\|\Delta_{A} v^{\prime}\right\|_{2}+\left(1+\varepsilon^{-1}\right)\left\|A_{a}\right\|_{\infty}^{2}\left\|v^{\prime}\right\|_{2} \\
& +\varepsilon\left\|\Delta v^{\prime}\right\|_{2}+C_{\varepsilon}\|B\|_{4}^{8}\left\|v^{\prime}\right\|_{2} .
\end{aligned}
$$

Taking $\varepsilon=1 / 3$ yields

$$
\left\|\Delta v^{\prime}\right\|_{2} \leq 2\left\|\Delta_{A} v^{\prime}\right\|_{2}+C\left(\left\|A_{a}\right\|_{\infty}^{2}+\|B\|_{4}^{8}\right)\left\|v^{\prime}\right\|_{2}
$$

and therefore by (2.38) (2.57)

$$
N_{5}^{\prime} \leq 4\left(N_{3}^{\prime}+r_{1}+M_{1}\right)+C\left(a^{2} T^{-2}+\widetilde{N}_{2}^{8} h(T)^{8}\right) N_{0}^{\prime}
$$

which is of the form

$$
N_{5}^{\prime} \leq 4\left(N_{3}^{\prime}+r_{1}+\left(o(1) ; \widetilde{N}_{2}, N_{0}, N_{0}^{\prime}, N_{1 / 2}\right)\right) .
$$


We finally estimate the Strichartz norms of $\nabla v^{\prime}$. For that purpose, by Lemma 2.1, we have to estimate the following quantity in the sum of spaces of the type $L^{\bar{q}}\left(J, L^{\bar{r}}\right)$ for admissible pairs $(q, r)$ :

$$
\begin{aligned}
Q & =\nabla\left(i A \cdot \nabla v^{\prime}+(1 / 2) A^{2} v^{\prime}+g\left(|u|^{2}\right) v^{\prime}+G_{1}-R_{1}\right) \\
& =i A \cdot \nabla^{2} v^{\prime}+i \nabla A \cdot \nabla_{A} v^{\prime}+(1 / 2) A^{2} \nabla v^{\prime}+\nabla\left(g\left(|u|^{2}\right) v^{\prime}\right)+\nabla G_{1}-\nabla R_{1} .
\end{aligned}
$$

The estimates are similar to those performed when estimating $\left\|v^{\prime}\right\|_{2}$ and the Strichartz norms of $v^{\prime}$ (see the proof of (2.42) (2.44)), with an additional gradient acting on each factor in each term, thereby producing the replacement of $N_{0}$ by $N_{1 / 2}$, of $N_{0}^{\prime}$ by $N_{1 / 2}^{\prime}$, of $N_{1 / 2}^{\prime}$ by $N_{5}^{\prime}$ and of $N_{2}$ by $N_{6}$ at suitable places. More precisely, the terms containing $v^{\prime}$ are estimated by

$$
\begin{aligned}
\left\|A_{a} \cdot \nabla^{2} v^{\prime}\right\|_{+} & \leq\|\| A_{a}\left\|_{\infty}\right\| \Delta v^{\prime}\left\|_{2}\right\|_{1} \leq 3 a N_{5}^{\prime} h(t), \\
\left\|\nabla A_{a} \cdot \nabla_{A} v^{\prime}\right\|_{+} & \leq\|\| A_{a}\left\|_{\infty}\right\| \nabla_{A} v^{\prime}\left\|_{2}\right\|_{1} \leq 3 a \widetilde{N}_{1 / 2}^{\prime} h(t), \\
\left\|A_{a}^{2} \nabla v^{\prime}\right\|_{+} & \leq\|\| A_{a}\left\|_{\infty}^{2}\right\| \nabla v^{\prime}\left\|_{2}\right\|_{1} \leq a^{2} N_{1 / 2}^{\prime} t^{-1} h(t), \\
\left\|B \cdot \nabla^{2} v^{\prime} ; L^{8 / 5}\left(J, L^{4 / 3}\right)\right\| & \leq\|\| B\left\|_{4}\right\| \Delta v^{\prime}\left\|_{2}\right\|_{8 / 5} \leq C N_{2} N_{5}^{\prime} h(t) \bar{h}(t), \\
\left\|\nabla B \cdot \nabla_{A} v^{\prime} ; L^{8 / 5}\left(J, L^{4 / 3}\right)\right\| & \leq\|\| B\left\|_{4}\right\| \nabla_{A} v^{\prime}\left\|_{2}\right\|_{8 / 5} \\
& \leq C N_{6} \widetilde{N}_{1 / 2}^{\prime} h(t) \bar{h}(t), \\
\left\|B^{2} \nabla v^{\prime} ; L^{2}\left(J, L^{6 / 5}\right)\right\| & \leq C\|\| B\left\|_{4}^{3 / 2}\right\| \nabla B\left\|_{4}^{1 / 2}\right\| \nabla v^{\prime}\left\|_{2}\right\|_{2} \\
& \leq C N_{2}^{3 / 2} N_{6}^{1 / 2} N_{1 / 2}^{\prime} h(t)^{3}, \\
\left\|\nabla\left(g\left(\left|u_{a}\right|^{2}\right) v^{\prime}\right)\right\|+ & \leq C c^{2}\left(N_{0}^{\prime}+N_{1 / 2}^{\prime}\right) h(t), \\
\left.\| \nabla\left(\bar{u}_{a} v\right) v^{\prime}\right) ; L^{4 / 3}\left(J, L^{3 / 2}\right) \| & \leq C c\left(N_{0} N_{0}^{\prime}+N_{0} N_{1 / 2}^{\prime}+N_{1 / 2} N_{0}^{\prime}\right) t^{1 / 4} h(t)^{2}, \\
\left.\| \nabla\left(|v|^{2}\right) v^{\prime}\right) ; L^{4 / 3}\left(J, L^{3 / 2}\right) \| & \leq C N_{1}\left(N_{0} N_{1 / 2}^{\prime}+N_{1 / 2} N_{0}^{\prime}\right) t^{1 / 2} h(t)^{3}
\end{aligned}
$$

where we have used again Lemmas 2.2 and 2.3 in the estimates of the terms containing $g$.

The terms from $\nabla G_{1}$ are estimated by

$$
\begin{aligned}
\left\|\nabla B \cdot \nabla_{A a} u_{a}\right\|_{+} & \leq N_{6}\left(C c_{4}+c a t^{-1}\right) h(t), \\
\left\|B \cdot \nabla \nabla_{A a} u_{a}\right\|_{+} & \leq C c N_{2}\left(1+a t^{-1}\right) h(t), \\
\left\|B^{2} \nabla u_{a}\right\|_{+} & \leq c N_{2}^{2} t^{-1} h(t)^{2}, \\
\left\|B(\nabla B) u_{a}\right\|_{+} & \leq c N_{2} N_{6} t^{-1} h(t)^{2} . \\
\left\|\nabla\left(g\left(\bar{u}_{a} v\right) u_{a}\right)\right\|_{+} & \leq C c_{3}\left(c_{3} N_{1 / 2}+c N_{0}\right) h(t), \\
\left\|\nabla\left(g\left(|v|^{2}\right) u_{a}\right)\right\|_{+} & \leq C N_{1}\left(c_{3} N_{1 / 2}+c N_{0}\right) t^{1 / 4} h(t)^{2} .
\end{aligned}
$$


Collecting the previous estimates yields

$$
\begin{aligned}
N_{4}^{\prime} \leq & C_{4}\left\{a\left(N_{5}^{\prime}+\tilde{N}_{1 / 2}^{\prime}\right)+a^{2} N_{1 / 2}^{\prime} T^{-1}+c\left(N_{6}+N_{2}\right)\left(1+a T^{-1}\right)\right. \\
& +c^{2}\left(N_{0}+N_{0}^{\prime}+N_{1 / 2}+N_{1 / 2}^{\prime}\right)+r_{1} \\
& +\left(N_{2} N_{5}^{\prime}+N_{6} \tilde{N}_{1 / 2}^{\prime}\right) \bar{h}(T)+c N_{2}\left(N_{6}+N_{2}\right) T^{-1} h(T) \\
& +c\left(\left(N_{1 / 2}+N_{0}\right)\left(N_{1}+N_{0}^{\prime}\right)+N_{0} N_{1 / 2}^{\prime}\right) T^{-1 / 8} \bar{h}(T) \\
& \left.+N_{2}^{3 / 2} N_{6}^{1 / 2} N_{1 / 2}^{\prime} h(T)^{2}+N_{1}\left(N_{0} N_{1 / 2}^{\prime}+N_{1 / 2} N_{0}^{\prime}\right) T^{-1 / 4} \bar{h}(T)^{2}\right\}
\end{aligned}
$$

which is of the form

$$
\begin{aligned}
N_{4}^{\prime} \leq & C_{4}\left(a\left(N_{5}^{\prime}+\tilde{N}_{1 / 2}^{\prime}\right)+c\left(N_{6}+N_{2}\right)+c^{2}\left(N_{0}+N_{0}^{\prime}+N_{1 / 2}+N_{1 / 2}^{\prime}\right)+r_{1}\right. \\
& \left.+\left(o(1) ; N_{0}, N_{0}^{\prime}, N_{1}, N_{1 / 2}, N_{1 / 2}^{\prime}, \widetilde{N}_{1 / 2}^{\prime}, N_{5}^{\prime}, N_{2}, N_{6}\right)\right) .
\end{aligned}
$$

From the previous estimates, more precisely from (2.42) (2.44) (2.46) (2.49) (2.54) (2.59) (2.62), it follows that the $N_{i}^{\prime}, 0 \leq i \leq 6$ are estimated in terms of the $N_{i}, 0 \leq i \leq 6$, provided $T$ is sufficiently large. In fact (2.42) (2.46) (2.49) provide estimates of $N_{0}^{\prime}, N_{2}^{\prime}$ and $N_{6}^{\prime}$. Denoting by $C$ a general constant depending on $T$ and on the $N_{i}$, it follows from (2.44) (2.59) and from the definition of $N_{1 / 2}^{\prime}, \tilde{N}_{1 / 2}^{\prime}$ that

$$
N_{1}^{\prime} \leq C\left(1+N_{1 / 2}^{\prime}\right), N_{1 / 2}^{\prime} \vee \widetilde{N}_{1 / 2}^{\prime} \leq C\left(1+N_{5}^{\prime}\right)^{1 / 2}, N_{5}^{\prime} \leq 4 N_{3}^{\prime}+C
$$

so that it remains only to estimate $N_{3}^{\prime}$ and $N_{4}^{\prime}$. Substituting the previous estimates into (2.54) (2.62) yields

$$
\left\{\begin{array}{l}
N_{3}^{\prime} \leq C_{3} N_{6} N_{4}^{\prime} \bar{h}(T)+\text { terms sublinear in } N_{3}^{\prime} \\
N_{4}^{\prime} \leq 4 C_{4} N_{3}^{\prime}\left(a+N_{2} \bar{h}(T)\right)+\text { terms sublinear in } N_{3}^{\prime}
\end{array}\right.
$$

which ensure the required estimate of $N_{3}^{\prime}, N_{4}^{\prime}$ provided $T$ is sufficiently large so that

$$
4 C_{3} C_{4}\left(a+N_{2} \bar{h}(T)\right) N_{6} \bar{h}(T)<1,
$$


which we assume from now on. Note that the terms responsible for that large $T$ condition are the terms $\partial_{t} B \cdot \nabla v^{\prime}$ from (2.51) and $A \cdot \nabla^{2} v^{\prime}$ from (2.61). No such condition was required at this stage in the simpler case of the $(\mathrm{WS})_{3}$ system [8]. The estimates obtained for the $N_{i}^{\prime}$ are obviously uniform in $t_{0}$.

We now take the limit $t_{0} \rightarrow \infty$ of $\left(v_{t_{0}}^{\prime}, B_{t_{0}}^{\prime}\right)$, restoring the subscript $t_{0}$ for that part of the argument. Let $T<t_{0}<t_{1}<\infty$ and let $\left(v_{t_{0}}^{\prime}, B_{t_{0}}^{\prime}\right)$ and $\left(v_{t_{1}}^{\prime}, B_{t_{1}}^{\prime}\right)$ be the corresponding solutions of (1.7). From the $L^{2}$ norm conservation of the difference $v_{t_{0}}^{\prime}-v_{t_{1}}^{\prime}$ and from (2.42), it follows that for all $t \in\left[T, t_{0}\right]$

$$
\left\|v_{t_{0}}^{\prime}(t)-v_{t_{1}}^{\prime}(t)\right\|_{2}=\left\|v_{t_{1}}^{\prime}\left(t_{0}\right)\right\|_{2} \leq K_{0} h\left(t_{0}\right)
$$

where $K_{0}$ is the RHS of (2.42), while from (1.7) (2.8) (2.9) (2.46) (2.49) and the initial conditions, it follows that

$$
\begin{aligned}
& \left\|B_{t_{0}}^{\prime}-B_{t_{1}}^{\prime} ; L^{4}\left(\left[T, t_{0}\right], W_{4}^{1}\right)\right\| \vee\left\|\partial_{t}\left(B_{t_{0}}^{\prime}-B_{t_{1}}^{\prime}\right) ; L^{4}\left(\left[T, t_{0}\right], L^{4}\right)\right\| \\
& \leq C\left\|G_{2}-R_{2} ; L^{4 / 3}\left(\left[t_{0}, t_{1}\right], W_{4 / 3}^{1}\right)\right\| \leq\left(K_{2}+K_{6}\right) h\left(t_{0}\right)
\end{aligned}
$$

where $K_{2}$ and $K_{6}$ are the RHS of (2.46) and (2.49) respectively.

It follows from $(2.67)(2.68)$ that there exists $\left(v^{\prime}, B^{\prime}\right) \in L_{l o c}^{\infty}\left([T, \infty), L^{2}\right) \oplus$ $L_{l o c}^{4}\left([T, \infty), W_{4}^{1}\right)$ with $\partial_{t} B^{\prime} \in L_{l o c}^{4}\left([T, \infty), L^{4}\right)$ such that $\left(v_{t_{0}}^{\prime}, B_{t_{0}}^{\prime}\right)$ converges to $\left(v^{\prime}, B^{\prime}\right)$ in that space when $t_{0} \rightarrow \infty$. From the uniformity in $t_{0}$ of the estimates (2.42) (2.46) (2.49), it follows that $\left(v^{\prime}, B^{\prime}\right)$ satisfies the same estimates in $[T, \infty)$, namely that (2.43) (2.47) (2.50) hold with $N_{i}^{\prime}$ defined by (2.33) (2.35) (2.39) with $I=[T, \infty)$. Furthermore it follows by a standard compactness argument that $\left(v^{\prime}, B^{\prime}\right) \in X([T, \infty))$ and that $v^{\prime}$ satisfies the remaining estimates, namely (2.45) (2.55) (2.60) (2.63) with the remaining $N_{i}^{\prime}$ again defined by $(2.34)(2.36)(2.37)(2.38)$ with $I=[T, \infty)$. Clearly $\left(v^{\prime}, B^{\prime}\right)$ satisfies the system (1.7).

From now on, $I$ denotes the interval $[T, \infty)$. The previous construction defines a map $\phi:(v, B) \rightarrow\left(v^{\prime}, B^{\prime}\right)$ from $X(I)$ to itself. The next step consists in proving that the map $\phi$ is a contraction on a suitable closed bounded set $\mathcal{R}$ of $X(I)$. We define $\mathcal{R}$ by the conditions (2.25)-(2.31) for some constants $N_{i}$ and for all $t \in I$. We first show that for a suitable choice of $N_{i}$ and for sufficiently large $T$, the map $\phi$ maps $\mathcal{R}$ into $\mathcal{R}$. By (2.43) (2.45) (2.47) (2.50) (2.55) (2.60) 
(2.63), it suffices for that purpose that

$$
\left\{\begin{array}{l}
\left(N_{0}^{\prime} \leq\right) C_{0}\left(c_{4} N_{2}+c_{3}^{2} N_{0}+r_{1}+o(1)\right) \leq N_{0} \\
\left(N_{1}^{\prime} \leq\right) C_{1}\left(c_{4} N_{2}+c_{3}^{2} N_{0}+c^{2} N_{0}^{\prime}+a N_{1 / 2}^{\prime}+r_{1}+o(1)\right) \leq N_{1} \\
\left(N_{2}^{\prime} \leq\right) C_{2}\left(c_{4} N_{0}+r_{2}+o(1)\right) \leq N_{2} \\
\left(N_{6}^{\prime} \leq\right) C_{6}\left(c_{4} N_{1 / 2}+r_{2}+o(1)\right) \leq N_{6} \\
\left(N_{3}^{\prime} \leq\right) C_{3}\left(a \tilde{N}_{1 / 2}^{\prime}+c_{4} N_{6}+c N_{2}+c_{3}^{2} N_{3}+c^{2}\left(N_{0}+N_{1}^{\prime}\right)+r_{1}+o(1)\right) \leq N_{3} \\
\left(N_{4}^{\prime} \leq\right) C_{4}\left(a\left(N_{5}^{\prime}+\tilde{N}_{1 / 2}^{\prime}\right)+c\left(N_{6}+N_{2}\right)+c^{2}\left(N_{0}+N_{0}^{\prime}+N_{1 / 2}+N_{1 / 2}^{\prime}\right)\right. \\
\left.\quad+r_{1}+o(1)\right) \leq N_{4} \\
\left(N_{5}^{\prime} \leq\right) 4\left(N_{3}^{\prime}+r_{1}+o(1)\right) \leq N_{5}
\end{array}\right.
$$

where we have omitted the dependence of the $o(1)$ terms on $N_{i}$ and $N_{i}^{\prime}$. We know in addition that

$$
N_{1 / 2}^{\prime} \vee \tilde{N}_{1 / 2}^{\prime} \leq 2\left(N_{0}^{\prime}\left(N_{3}^{\prime}+r_{1}+o(1)\right)\right)^{1 / 2} .
$$

In order to ensure (2.69), we proceed as follows. We first choose $N_{0}$ and $N_{2}$ by imposing

$$
\left\{\begin{array}{l}
N_{0}=C_{0}\left(c_{4} N_{2}+c_{3}^{2} N_{0}+r_{1}+1\right) \\
N_{2}=C_{2}\left(c_{4} N_{0}+r_{2}+1\right)
\end{array}\right.
$$

which is possible under the smallness condition on $c_{3}, c_{4}$

$$
C_{0}\left(C_{2} c_{4}^{2}+c_{3}^{2}\right)<1,
$$

and we impose the condition that $o(1) \leq 1$ in (2.43) (2.47) by taking $T$ sufficiently large (depending on $N_{0}, N_{2}$ just chosen and on $N_{1 / 2}, N_{1}$ to be chosen later). This ensures the $N_{0}^{\prime} \leq N_{0}$ and $N_{2}^{\prime} \leq N_{2}$ parts of (2.69). Furthermore one can replace $N_{0}^{\prime}$ by $N_{0}$ in all the remaining estimates. We next impose

$$
\left\{\begin{array}{l}
N_{5}=4\left(N_{3}+r_{1}+1\right) \\
N_{6}=C_{6}\left(c_{4}\left(N_{0} N_{5}\right)^{1 / 2}+r_{2}+1\right)=C_{6}\left(2 c_{4}\left(N_{0}\left(N_{3}+r_{1}+1\right)\right)^{1 / 2}+r_{2}+1\right)
\end{array}\right.
$$

and we impose $o(1) \leq 1$ in (2.50) and (2.60) by taking $T$ sufficiently large depending on the relevant $N_{i}$. This ensures the $N_{6}^{\prime} \leq N_{6}$ part of (2.69) together with the inequality

$$
N_{5}^{\prime} \leq 4\left(N_{3}^{\prime}+r_{1}+1\right)
$$


which will ensure the $N_{5}^{\prime} \leq N_{5}$ part of (2.69) as soon as the $N_{3}^{\prime} \leq N_{3}$ part holds. Furthermore, under the choices and assumptions made so far, (2.74) implies

$$
N_{1 / 2}^{\prime} \vee \tilde{N}_{1 / 2}^{\prime} \leq 2\left(N_{0}\left(N_{3}^{\prime}+r_{1}+1\right)\right)^{1 / 2}
$$

We now substitute (2.75) into (2.44), we substitute (2.74) (2.75) into (2.62) and we substitute the results and (2.75) again into (2.54), thereby obtaining an estimate of the form

$$
\begin{aligned}
N_{3}^{\prime} \leq & f\left(N_{3}^{\prime},\left\{N_{i}\right\}\right) \equiv C_{3}\left(2 a\left(N_{0}\left(N_{3}^{\prime}+r_{1}+1\right)\right)^{1 / 2}\right. \\
& +c_{4} C_{6}\left(2 c_{4}\left(N_{0}\left(N_{3}+r_{1}+1\right)\right)^{1 / 2}+r_{2}+1\right) \\
& \left.+c N_{2}+c_{3}^{2} N_{3}+2 c^{2} N_{0}+r_{1}+o(1)\right)
\end{aligned}
$$

where $f\left(N_{3}^{\prime},\left\{N_{i}\right\}\right)$ is a positive increasing concave function of $N_{3}^{\prime}$ for fixed $T$ and $N_{i}$. It follows therefrom that (2.76) will imply $N_{3}^{\prime} \leq N_{3}$ provided we ensure that

$$
N_{3} \geq f\left(N_{3},\left\{N_{i}\right\}\right)
$$

This is obtained by imposing

$$
\begin{aligned}
N_{3}= & C_{3}\left(2\left(a+C_{6} c_{4}^{2}\right)\left(N_{0}\left(N_{3}+r_{1}+1\right)\right)^{1 / 2}+c_{4} C_{6}\left(r_{2}+1\right)\right. \\
& \left.+c N_{2}+c_{3}^{2} N_{3}+2 c^{2} N_{0}+r_{1}+1\right)
\end{aligned}
$$

which is possible under the smallness condition $C_{3} c_{3}^{2}<1$, and by imposing that $o(1) \leq 1$ in $(2.76)$ by taking $T$ sufficiently large depending on the $N_{i}$.

It is then a simple matter to choose $N_{1}$ and $N_{4}$ in order to ensure the $N_{1}^{\prime} \leq N_{1}$ and $N_{4}^{\prime} \leq N_{4}$ parts of (2.69), since all the $N_{i}^{\prime}$ in the RHS of (2.44) and (2.62) are now under control. It suffices to choose

$$
\begin{aligned}
& N_{1}=C_{1}\left(c_{4} N_{2}+2 c^{2} N_{0}+a\left(N_{0} N_{5}\right)^{1 / 2}+r_{1}+1\right) \\
& N_{4}=C_{4}\left(a N_{5}+\left(a+2 c^{2}\right)\left(N_{0} N_{5}\right)^{1 / 2}+c\left(N_{6}+N_{2}\right)+2 c^{2} N_{0}+r_{1}+1\right)
\end{aligned}
$$

and to impose that $o(1) \leq 1$ in (2.45) (2.63) by taking $T$ sufficiently large depending on the $N_{i}$ (with the $N_{i}^{\prime}$ in the $o(1)$ terms being estimated by the $\left.N_{i}\right)$.

We now show that the map $\phi$ is a contraction on $\mathcal{R}$ for a suitable norm defined on $X(I)$. Let $\left(v_{i}, B_{i}\right) \in \mathcal{R}$ and $\left(v_{i}^{\prime}, B_{i}^{\prime}\right)=\phi\left(\left(v_{i}, B_{i}\right)\right), i=1,2$. For any 
pair of functions $f_{1}, f_{2}$, we define $f_{ \pm}=(1 / 2)\left(f_{1} \pm f_{2}\right)$ so that $f_{1}=f_{+}+f_{-}$, $f_{2}=f_{+}-f_{-}$and $(f g)_{ \pm}=f_{+} g_{ \pm}+f_{-} g_{\mp}$. In particular $u_{+}=u_{a}+v_{+}, u_{-}=v_{-}$, $A_{+}=A_{a}+B_{+}, A_{-}=B_{-},\left(\Delta_{A}\right)_{-}=-2 i B_{-} \cdot \nabla_{A_{+}}$, and similarly for the primed quantities. Since $\mathcal{R}$ is convex and stable under $\phi,\left(v_{+}, B_{+}\right)$and $\left(v_{+}^{\prime}, B_{+}^{\prime}\right)$ belong to $\mathcal{R}$, namely satisfy $(2.25)-(2.31)$. Corresponding to $(1.7),\left(v_{-}^{\prime}, B_{-}^{\prime}\right)$ satisfies the system

$$
\left\{\begin{array}{c}
i \partial_{t} v_{-}^{\prime}=-(1 / 2)\left(\Delta_{A}\right)_{+} v_{-}^{\prime}+g\left(|u|_{+}^{2}\right) v_{-}^{\prime}+i B_{-} \cdot \nabla_{A_{+}}\left(u_{a}+v_{+}^{\prime}\right) \\
\quad+g\left(2 \operatorname{Re}\left(\bar{u}_{a}+\bar{v}_{+}\right) v_{-}\right)\left(u_{a}+v_{+}^{\prime}\right) \\
\square B_{-}^{\prime}=2 P \operatorname{Im}\left(\bar{v}_{-} \nabla_{A_{+}}\left(u_{a}+v_{+}\right)\right)-P B_{-}\left(\left|u_{a}+v_{+}\right|^{2}+\left|v_{-}\right|^{2}\right) .
\end{array}\right.
$$

Here however, in contrast with the case of the $(\mathrm{WS})_{3}$ system where the corresponding map $\phi$ can be shown to be a contraction for the whole norm of $X(I)$, we encounter a difficulty due to the derivative coupling in the covariant Laplacian. In fact if $D$ is a differential operator of order $m$, a straightforward energy estimate of $\left\|D v_{-}^{\prime}\right\|_{2}$ from (2.81) yields

$$
\partial_{t}\left\|D v_{-}^{\prime}\right\|_{2} \leq\left\|B_{-} \cdot D \nabla_{A_{+}} v_{+}^{\prime}\right\|_{2}+\text { other terms }
$$

and requires therefore a control of $v_{+}^{\prime}$ at order $m+1$, so that one can hope to contract norms of $v$ of degree at most one less than those occurring in the definition of $X(I)$. Fortunately, because of the special algebraic properties of the equations, it turns out that the lowest two semi norms of $X(I)$ for the differences, namely those corresponding to $N_{0}$ and $N_{2}$, can be decoupled from the higher ones and can be contracted on the bounded sets of $X(I)$. This follows from the fact that the symmetry of the quadratic form $P \operatorname{Im}\left(\bar{v}_{1} \nabla_{A} v_{2}\right)$ has made it possible to avoid having a gradient acting on $v_{-}$in the equation for $B_{-}^{\prime}$ in (2.81). Thus we shall show that $\phi$ is a contraction for the pair of semi norms

$$
\left\{\begin{array}{l}
N_{0}=\operatorname{Sup}_{t \in I} h(t)^{-1}\|v(t)\|_{2} \\
N_{2}=\operatorname{Sup}_{t \in I} h(t)^{-1}\left\|B ; L^{4}\left([t, \infty), L^{4}\right)\right\|
\end{array}\right.
$$

Let $\left(N_{0_{-}}, N_{2_{-}}\right)$and $\left(N_{0-}^{\prime}, N_{2-}^{\prime}\right)$ be the corresponding semi norms of $\left(v_{-}, B_{-}\right)$ and $\left(v_{-}^{\prime}, B_{-}^{\prime}\right)$ respectively. We have to estimate $\left(N_{0_{-}}^{\prime}, N_{2-}^{\prime}\right)$ in terms of $\left(N_{0_{-}}\right.$, $N_{2-}$ ). We first estimate $N_{0-}^{\prime}$. From $(2.81)$ we obtain

$$
\left\|v_{-}^{\prime}(t)\right\|_{2} \leq\left\|B_{-} \cdot \nabla_{A_{+}}\left(u_{a}+v_{+}^{\prime}\right)\right\|_{+}+\left\|g\left(2 \operatorname{Re}\left(\bar{u}_{a}+\bar{v}_{+}\right) v_{-}\right)\left(u_{a}+v_{+}^{\prime}\right)\right\|_{+} .
$$


The terms not containing $v_{+}^{\prime}$ are estimated as in the proof of (2.42), namely

$$
\begin{aligned}
\left\|B_{-} \cdot \nabla u_{a}\right\|_{+} & \leq C c_{4} N_{2-} h(t), \\
\left\|B_{-} \cdot A_{a} u_{a}\right\|_{+} & \leq c a N_{2-} t^{-1} h(t), \\
\left\|B_{-} B_{+} u_{a}\right\|_{+} & \leq c N_{2-} N_{2} t^{-1} h(t)^{2}, \\
\left\|g\left(\bar{u}_{a} v_{-}\right) u_{a}\right\|_{+} & \leq C c_{3}^{2} N_{0-} h(t), \\
\left\|g\left(\bar{v}_{+} v_{-}\right) u_{a}\right\|_{+} & \leq C c_{3} N_{0-} N_{1} t^{1 / 4} h(t)^{2} .
\end{aligned}
$$

We next estimate the terms containing $v_{+}^{\prime}$.

$$
\left\|B_{-} \cdot \nabla v_{+}^{\prime}\right\|_{+} \leq\|\| B_{-}\left\|_{4}\right\| \nabla v_{+}^{\prime}\left\|_{4}\right\|_{1} \leq C N_{2-} N_{4} h(t) \bar{h}(t)
$$

by Lemma 2.2 ,

$$
\begin{aligned}
\left\|B_{-} \cdot A_{a} v_{+}^{\prime}\right\|_{+} & \leq\|\| B_{-}\left\|_{4}\right\| A_{a}\left\|_{\infty}\right\| v_{+}^{\prime}\left\|_{4}\right\|_{1} \\
& \leq a N_{2-} N_{1} h^{2}\left\|t^{-1}\right\|_{8 / 3} \leq a N_{2-} N_{1} t^{-5 / 8} h(t)^{2} \\
\left\|B_{-} B_{+} v_{+}^{\prime}\right\|_{+} & \leq C\|\| B_{-}\left\|_{4}\left(\left\|B_{+}\right\|_{4}\left\|\nabla B_{+}\right\|_{4}^{3}\right)^{1 / 4}\right\| v_{+}^{\prime}\left\|_{4}\right\|_{1} \\
& \leq C N_{2-}\left(N_{2} N_{6}^{3}\right)^{1 / 4} N_{1} t^{1 / 8} h(t)^{3}
\end{aligned}
$$

by Lemma 2.2,

$$
\begin{aligned}
& \left\|g\left(\bar{u}_{a} v_{-}\right) v_{+}^{\prime}\right\|_{+} \leq C\|\| u_{a}\left\|_{3}\right\| v_{-}\left\|_{2}\right\| v_{+}^{\prime}\left\|_{3}\right\|_{1} \leq C c_{3} N_{0-} N_{1} t^{1 / 4} h(t)^{2}, \\
& \left\|g\left(\bar{v}_{+} v_{-}\right) v_{+}^{\prime}\right\|_{+} \leq C\|\| v_{+}\left\|_{3}\right\| v_{-}\left\|_{2}\right\| v_{+}^{\prime}\left\|_{3}\right\|_{1} \leq C N_{0-} N_{1}^{2} t^{1 / 2} h(t)^{3}
\end{aligned}
$$

by Lemma 2.3, part (1) and Lemma 2.2 .

Collecting the previous estimates yields

$$
\begin{aligned}
N_{0-}^{\prime} & \leq C_{0}\left\{N _ { 2 - } \left(c_{4}+a c T^{-1}+c N_{2} T^{-1} h(T)+N_{4} \bar{h}(T)+a N_{1} T^{-5 / 8} h(T)\right.\right. \\
& \left.+\left(N_{2} N_{6}^{3}\right)^{1 / 4} N_{1} T^{-1 / 4} h(T) \bar{h}(T)\right)+N_{0-}\left(c_{3}^{2}+c N_{1} T^{-1 / 8} \bar{h}(T)\right. \\
& \left.\left.+N_{1}^{2} T^{-1 / 4} \bar{h}(T)^{2}\right)\right\}
\end{aligned}
$$

which is of the form

$$
N_{0-}^{\prime} \leq C_{0}\left(N_{2-}\left(c_{4}+o(1)\right)+N_{0-}\left(c_{3}^{2}+o(1)\right)\right) .
$$

We next estimate $N_{2-}^{\prime}$. From (2.8) (2.81) we obtain

$$
\left\|B_{-}^{\prime} ; L^{4}\left(J, L^{4}\right)\right\| \leq C\left(\left\|\bar{v}_{-} \nabla_{A_{+}}\left(u_{a}+v_{+}\right)\right\|_{*}+\left\|B_{-}\left(\left|u_{a}\right|^{2}+\left|v_{+}\right|^{2}\right)\right\|_{*}\right) .
$$


The linear terms are estimated as in the proof of (2.46), namely

$$
\begin{aligned}
\left\|\bar{v}_{-} \nabla u_{a}\right\|_{*} & \leq 2 c_{4} N_{0-} h(t), \\
\left\|\bar{v}_{-} A_{a} u_{a}\right\|_{*} & \leq a c N_{0-} t^{-1} h(t), \\
\left\|B_{-}\left|u_{a}\right|^{2}\right\|_{*} & \leq c^{2} N_{2-} t^{-1} h(t) .
\end{aligned}
$$

The non linear terms are estimated in a slightly different way. The quadratic terms are estimated by

$$
\begin{aligned}
\left\|\bar{v}_{-} \nabla v_{+}\right\|_{*} & \leq\|\| v_{-}\left\|_{2}\right\| \nabla v_{+}\left\|_{4}\right\|_{4 / 3} \leq C N_{0-} N_{4} h(t) \bar{h}(t), \\
\left\|\bar{v}_{-} A_{a} v_{+}\right\|_{*} & \leq\|\| A_{a}\left\|_{\infty}\right\| v_{-}\left\|_{2}\right\| v_{+}\left\|_{4}\right\|_{4 / 3} \leq a N_{0-} N_{1} t^{-5 / 8} h(t)^{2}, \\
\left\|\bar{v}_{-} B_{+} u_{a}\right\|_{*} & \leq\|\| v_{-}\left\|_{2}\right\| B_{+}\left\|_{4}\right\| u_{a}\left\|_{\infty}\right\|_{4 / 3} \\
& \leq c N_{0-} N_{2} h\left\|t^{-3 / 2} h\right\|_{2} \leq c N_{0-} N_{2} t^{-1} h(t)^{2} .
\end{aligned}
$$

The cubic terms are estimated by

$$
\begin{aligned}
\left\|\bar{v}_{-} B_{+} v_{+}\right\|_{*} & \leq C\|\| v_{-}\left\|_{2}\left(\left\|B_{+}\right\|_{4}\left\|\nabla B_{+}\right\|_{4}^{3}\right)^{1 / 4}\right\| v_{+}\left\|_{4}\right\|_{4 / 3} \\
& \leq C N_{0-}\left(N_{2} N_{6}^{3}\right)^{1 / 4} N_{1} t^{1 / 8} h(t)^{3}
\end{aligned}
$$

by Lemma 2.2 ,

$$
\left\|B_{-}\left|v_{+}\right|^{2}\right\|_{*} \leq C N_{2-} N_{1}^{3 / 2} N_{1 / 2}^{1 / 2} h(t)^{3} .
$$

Collecting the previous estimates yields

$$
\begin{aligned}
N_{2-}^{\prime} \leq & C_{2}\left\{N _ { 0 - } \left(c_{4}+a c T^{-1}+N_{4} \bar{h}(T)+a N_{1} T^{-5 / 8} h(T)+c N_{2} T^{-1} h(T)\right.\right. \\
& \left.\left.+\left(N_{2} N_{6}^{3}\right)^{1 / 4} N_{1} T^{-1 / 4} h(T) \bar{h}(T)\right)+N_{2-}\left(c^{2} T^{-1}+N_{1}^{3 / 2} N_{1 / 2}^{1 / 2} h(T)^{2}\right)\right\}
\end{aligned}
$$

which is of the form

$$
N_{2-}^{\prime} \leq C_{2}\left(c_{4}+o(1)\right) N_{0-}+o(1) N_{2-} .
$$

It follows from (2.85) (2.88) that the map $\phi$ is a contraction for the pair of semi norms $\left(N_{0}, N_{2}\right)$ on the set $\mathcal{R}$ under the smallness condition

$$
C_{0}\left(C_{2} c_{4}^{2}+c_{3}^{2}\right)<1
$$

and for $T$ sufficiently large. Since the set $\mathcal{R}$ is closed for the norm defined by the pair $\left(N_{0}, N_{2}\right)$, it follows therefrom that the system (1.4) has a solution in 
$\mathcal{R}$. This proves the existence part of Proposition 2.2. The uniqueness part follows from (2.85) (2.88) again with $N_{i-}^{\prime}=N_{i-}$.

We remark at this point that the constants $C_{0}, C_{2}$ appearing in (2.89) can be taken to be the same as in (2.72) so that the two smallness conditions actually coincide. In fact those constants are determined by the linear terms in the estimates, and those terms are the same in both cases. There may occur additional, different constants coming from the non linear terms. They have been omitted in (2.84) (2.87).

It remains to prove the last statement of Proposition 2.2 and for that purpose we need to estimate the energy norm of $B^{\prime}$. From (1.7) (2.10) it follows that for all $t \in I$

$$
\left\|\nabla B^{\prime}(t)\right\|_{2} \vee\left\|\partial_{t} B^{\prime}(t)\right\|_{2} \leq\left\|G_{2}-R_{2}\right\|_{+}
$$

where $G_{2}$ is defined by (1.5). We estimate the various terms of $G_{2}$ successively. The linear terms in $v$ are estimated by

$$
\begin{aligned}
\left\|\bar{v} \nabla u_{a}\right\|_{+} & \leq\|\| v\left\|_{2}\right\| \nabla u_{a}\left\|_{\infty}\right\|_{1} \\
& \leq c N_{0}\left\|t^{-3 / 2} h\right\|_{1} \leq 2 c N_{0} t^{-1 / 2} h(t), \\
\left\|\bar{v} A_{a} u_{a}\right\|_{+} & \leq\|\| v\left\|_{2}\right\| A_{a}\left\|_{\infty}\right\| u_{a}\left\|_{\infty}\right\|_{1} \\
& \leq a c N_{0}\left\|t^{-5 / 2} h\right\|_{1} \leq a c N_{0} t^{-3 / 2} h(t) .
\end{aligned}
$$

The linear term in $B$ is estimated by

$$
\begin{aligned}
\left\|B\left|u_{a}\right|^{2}\right\|_{+} & \leq\|\| B\left\|_{4}\right\| u_{a}\left\|_{4}\right\| u_{a}\left\|_{\infty}\right\|_{1} \\
& \leq c^{2} N_{2} h\left\|t^{-3 / 4-3 / 2}\right\|_{4 / 3} \leq c^{2} N_{2} t^{-3 / 2} h(t) .
\end{aligned}
$$

The quadratic terms in $v^{2}$ are estimated by

$$
\|\bar{v} \nabla v\|_{+} \leq\|\| v\left\|_{4}\right\| \nabla v\left\|_{4}\right\|_{1} \leq C N_{1} N_{4} t^{1 / 4} h(t)^{2}
$$

by Lemma 2.2 ,

$$
\begin{aligned}
\left\|A_{a}|v|^{2}\right\|_{+} & \leq\|\| A_{a}\left\|_{\infty}\right\| v\left\|_{4}^{2}\right\|_{1} \\
& \leq a N_{1}^{2} h^{2}\left\|t^{-1}\right\|_{4} \leq a N_{1}^{2} t^{-3 / 4} h(t)^{2} .
\end{aligned}
$$

The quadratic terms in $B v$ again need not be considered. The cubic term $B|v|^{2}$ is estimated by

$$
\begin{aligned}
\left\|B|v|^{2}\right\|_{+} & \leq C\left\|B ; L^{4}\left(L^{4}\right)\right\|\left\|v ; L^{8 / 3}\left(L^{4}\right)\right\|^{5 / 4}\left\|\nabla v ; L^{8 / 3}\left(L^{4}\right)\right\|^{3 / 4} \\
& \leq C N_{2} N_{1}^{5 / 4} N_{4}^{3 / 4} h(t)^{3} .
\end{aligned}
$$


Collecting the previous estimates and using (2.23), we obtain

$$
\begin{aligned}
& \left\|\nabla B^{\prime}(t)\right\|_{2} \vee\left\|\partial_{t} B^{\prime}(t)\right\|_{2} \leq C\left(c N_{0} t^{-1 / 2}+a c N_{0} t^{-3 / 2}+c^{2} N_{2} t^{-3 / 2}\right. \\
& \left.+N_{1} N_{4} t^{1 / 4} h(t)+a N_{1}^{2} t^{-3 / 4} h(t)+N_{2} N_{1}^{5 / 4} N_{4}^{3 / 4} h(t)^{2}+r_{2} t^{-1 / 2}\right) h(t)
\end{aligned}
$$

which proves that the solution of (1.4) constructed previously satisfies (2.24).

Remark 2.2. The only smallness condition on $u$ is the condition (2.72), coming from $N_{0}$ and from its coupling with $N_{2}$. The subsequent condition $C_{3} c_{3}^{2}<1$ needed for the choice of $N_{3}$ comes in fact from exactly the same estimate as the $c_{3}^{2}$ contribution to $N_{0}^{\prime}$, so that the latter condition is actually the $c_{4}=0$ special case of $(2.72)$ and is therefore weaker than (2.72). That fact is hidden by the use of overall constants $C_{0}$ and $C_{3}$ in the estimates of $N_{0}^{\prime}$ and $N_{3}^{\prime}$.

\section{§3. Remainder Estimates and Completion of the Proof}

In this section, we first prove that the choice of asymptotic functions $\left(u_{a}, A_{a}\right)$ made in the introduction satisfies the assumptions of Proposition 2.2 for the choice of $h$ made in Proposition 1.1, under suitable assumptions on the asymptotic state $\left(u_{+}, A_{+}, \dot{A}_{+}\right)$. We then combine those results with Proposition 2.2 to complete the proof of Proposition 1.1.

We first supplement the definition of $\left(u_{a}, A_{a}\right)$ with some additional properties of a general character. In addition to the representation (1.13) (1.14) of $A_{1}$, we need a representation of $\partial_{t} A_{1}$. From (1.12) it follows that

$$
\partial_{t} A_{1}(t)=-\int_{t}^{\infty} d t^{\prime} \cos \left(\omega\left(t^{\prime}-t\right)\right) t^{\prime-1} P x\left|u_{a}\left(t^{\prime}\right)\right|^{2}
$$

so that upon substitution of (1.8) we obtain

$$
\partial_{t} A_{1}(t)=t^{-2} D_{0}(t) \widetilde{\widetilde{A}}_{1}
$$

where

$$
\widetilde{\widetilde{A}}_{1}=-\int_{t}^{\infty} d \nu \nu^{-3} \cos (\omega(\nu-1)) D_{0}(\nu) P x\left|w_{+}\right|^{2} .
$$

On the other hand, from (1.13)

$$
\nabla A_{1}(t)=t^{-2} D_{0}(t) \nabla \widetilde{A}_{1} .
$$


We shall need the operator

$$
J \equiv J(t)=x+i t \nabla
$$

The asymptotic form $A_{a}$ for $A$ has been chosen in order to make $R_{2}$ small. In fact $R_{2}$ can be rewritten as

$$
R_{2}=\square A_{a}+P\left(t^{-1} \operatorname{Re} \bar{u}_{a} J u_{a}+\left(A_{a}-x / t\right)\left|u_{a}\right|^{2}\right)
$$

and $A_{a}$ has been chosen in such a way that

$$
\square A_{a}=P(x / t)\left|u_{a}\right|^{2}
$$

so that

$$
R_{2}=P\left(t^{-1} \operatorname{Re} \bar{u}_{a} J u_{a}+A_{a}\left|u_{a}\right|^{2}\right) .
$$

Under general assumptions on $\left(u_{a}, A_{a}\right)$, of the same type as in Proposition 2.2 (see especially (2.17) (2.20)) but not making use of their special form, we can prove that $R_{2}$ satisfies the assumptions needed for that proposition with the choice of $h$ required for Proposition 1.1.

Lemma 3.1. Let $\left(u_{a}, A_{a}\right)$ satisfy the estimates

$$
\begin{aligned}
\left\|u_{a}(t)\right\|_{r} & \leq c t^{-\delta(r) \quad \text { for } 2 \leq r \leq \infty}, \\
\left\|\nabla u_{a}(t)\right\|_{4} & \leq c t^{-3 / 4} \\
\left\|J u_{a}(t)\right\|_{2} & \leq c_{1}(1+\ell n t) \\
\left\|A_{a}(t)\right\|_{\infty} & \vee\left\|\nabla A_{a}(t)\right\|_{\infty} \leq a t^{-1}
\end{aligned}
$$

for all $t \geq 1$. Then $R_{2}$ satisfies the estimates

$$
\begin{aligned}
\left\|R_{2} ; L^{4 / 3}\left([t, \infty), L^{4 / 3}\right)\right\| & \vee\left\|\nabla R_{2} ; L^{4 / 3}\left([t, \infty), L^{4 / 3}\right)\right\| \\
& \leq r_{2} t^{-1}(1+\ell n t), \\
\left\|R_{2} ; L^{1}\left([t, \infty), L^{2}\right)\right\| & \leq r_{2} t^{-3 / 2}(1+\ell n t)
\end{aligned}
$$

for some constant $r_{2}$ and for all $t \geq 1$.

Proof. We estimate

$$
\begin{aligned}
\left\|R_{2}(t)\right\|_{4 / 3} & \leq C\left\|u_{a}\right\|_{4}\left(t^{-1}\left\|J u_{a}\right\|_{2}+\left\|A_{a}\right\|_{\infty}\left\|u_{a}\right\|_{2}\right) \\
& \leq C t^{-7 / 4} c\left(c_{1}(1+\ell n t)+a c\right)
\end{aligned}
$$


which implies the first estimate of (3.13) by integration,

$$
\begin{aligned}
\left\|R_{2}(t)\right\|_{2} & \leq\left\|u_{a}\right\|_{\infty}\left(t^{-1}\left\|J u_{a}\right\|_{2}+\left\|A_{a}\right\|_{\infty}\left\|u_{a}\right\|_{2}\right) \\
& \leq t^{-5 / 2} c\left(c_{1}(1+\ell n t)+a c\right)
\end{aligned}
$$

which implies (3.14) by integration.

In order to prove the second estimate of (3.13), we note that the quadratic form

$$
P \operatorname{Re}\left(t^{-1} \bar{v}_{1} J v_{2}+A_{a} \bar{v}_{1} v_{2}\right)
$$

is symmetric in $v_{1}, v_{2}$, so that

$$
\begin{aligned}
\nabla R_{2}= & 2 P \operatorname{Re}\left(t^{-1}\left(\nabla \bar{u}_{a}\right) J u_{a}+A_{a}\left(\nabla \bar{u}_{a}\right) u_{a}\right) \\
& +P\left(\nabla\left(A_{a}+x / t\right)\right)\left|u_{a}\right|^{2}
\end{aligned}
$$

and therefore

$$
\begin{aligned}
\left\|\nabla R_{2}\right\|_{4 / 3} \leq & C\left(\left\|\nabla u_{a}\right\|_{4}\left(t^{-1}\left\|J u_{a}\right\|_{2}+\left\|A_{a}\right\|_{\infty}\left\|u_{a}\right\|_{2}\right)\right. \\
& \left.+\left(\left\|\nabla A_{a}\right\|_{\infty}+t^{-1}\right)\left\|u_{a}\right\|_{4}\left\|u_{a}\right\|_{2}\right) \\
\leq & C t^{-7 / 4}\left(c\left(c_{1}(1+\ell n t)+a c\right)+c^{2}(a+1)\right)
\end{aligned}
$$

from which the second estimate of (3.13) follows by integration.

We now turn to $R_{1}$. We first skim $R_{1}$ of some harmless terms. Expanding the covariant Laplacian and using again $J$, we rewrite $R_{1}$ as

$$
R_{1}=R_{1,1}+R_{1,2}
$$

where

$$
\begin{aligned}
& R_{1,1}=i \partial_{t} u_{a}+(1 / 2) \Delta u_{a}+t^{-1}\left(x \cdot A_{1}\right) u_{a}-g\left(\left|u_{a}\right|^{2}\right) u_{a}, \\
& R_{1,2}=t^{-1}\left(x \cdot A_{0}\right) u_{a}-t^{-1} A_{a} \cdot J u_{a}-(1 / 2) A_{a}^{2} u_{a} .
\end{aligned}
$$

In the same way as for $R_{2}$, we can show that $R_{1,2}$ satisfies the assumptions needed for Proposition 2.2 with the choice of $h$ required for Proposition 1.1 under general assumptions on $\left(u_{a}, A_{a}\right)$ not making use of their special form.

Lemma 3.2. Let $u_{a}, A_{a}$ and $A_{0}$ satisfy the estimates

$$
\begin{aligned}
& \left\|\partial_{t}^{j} \nabla^{k} u_{a}\right\|_{2} \leq c, \\
& \left\|\partial_{t}^{j} \nabla^{k} J u_{a}\right\|_{2} \leq c_{1}(1+\ln t), \\
& \left\|\partial_{t}^{j} \nabla^{k} A_{a}\right\|_{\infty} \leq a t^{-1} \\
& \left\|\partial_{t}^{j} \nabla^{k}\left(x \cdot A_{0}\right)\right\|_{\infty} \leq a_{0} t^{-1},
\end{aligned}
$$


for $0 \leq j+k \leq 1$ and for all $t \geq 1$. Then $R_{1,2}$ satisfies the estimates

$$
\left\|\partial_{t}^{j} \nabla^{k} R_{1,2}\right\|_{2} \leq r_{1,2} t^{-2}(1+\ln t)
$$

for $0 \leq j+k \leq 1$, for some constant $r_{1,2}$ and for all $t \geq 1$.

Proof. We estimate

$$
\begin{aligned}
\left\|R_{1,2}\right\|_{2} & \leq t^{-2}\left(\left(a_{0}+(1 / 2) a^{2}\right) c+a c_{1}(1+\ell n t)\right), \\
\left\|\nabla R_{1,2}\right\|_{2} & \leq t^{-2}\left(\left(2 a_{0}+(3 / 2) a^{2}\right) c+2 a c_{1}(1+\ell n t)\right), \\
\left\|\partial_{t} R_{1,2}\right\|_{2} & \leq \operatorname{idem}+t^{-3}\left(\left(a_{0}+a c_{1}(1+\ell n t)\right) .\right.
\end{aligned}
$$

We now turn to $R_{1,1}$. We shall need the commutation relations

$$
\begin{aligned}
& \nabla M D=M D\left(i x+t^{-1} \nabla\right) \equiv M D \widetilde{\nabla} \\
& i \partial_{t} M D=M D\left(i \partial_{t}+(1 / 2) x^{2}-i t^{-1}(x \cdot \nabla+3 / 2)\right) \equiv M D i \widetilde{\partial}_{t} \\
& J M D=i M D \nabla \\
& \left(i \partial_{t}+(1 / 2) \Delta\right) M D=M D\left(i \partial_{t}+\left(2 t^{2}\right)^{-1} \Delta\right) .
\end{aligned}
$$

In particular $(3.23)(3.24)$ are taken as the definitions of $\widetilde{\nabla}$ and $\widetilde{\partial}_{t}$. From the choice (1.8) of $u_{a}$ and from (3.26), it follows that

$$
R_{1,1}=M D\left(i \partial_{t}+\left(2 t^{2}\right)^{-1} \Delta+t^{-1} x \cdot \widetilde{A}_{1}-t^{-1} g\left(\left|w_{+}\right|^{2}\right)\right) \exp (-i \varphi) w_{+}
$$

The choice (1.15) of $\varphi$ has been taylored to cancel the two long range terms in (3.27), so that

$$
R_{1,1}=\left(2 t^{2}\right)^{-1} M D \Delta \exp (-i \varphi) w_{+}
$$

We now have to prove that the previous choice of $\left(u_{a}, A_{a}\right)$ satisfies the remaining assumptions of Proposition 2.2 and of Lemmas 3.1 and 3.2. More precisely we have to prove that $\left(u_{a}, A_{a}\right)$ satisfies the estimates $(2.17)(2.19)$ (2.20) (3.19) (3.21) and the analogue of (3.22) for $R_{1,1}$. (Note that (3.9) (3.10) (3.18) are special cases of (2.17) and that (3.12) is a special case of (3.20) which is identical with (2.20)).

The contribution of $A_{0}$ to $A_{a}$ and to $R_{1,2}$ will be taken care of by the following general estimates of solutions of the wave equation. 
Lemma 3.3. Let $A_{0}$ be defined by (1.11) and let $k \geq 0$ be an integer. Let $A_{+}$and $\dot{A}_{+}$satisfy the conditions

$$
\nabla^{2} A_{+}, \nabla \dot{A}_{+} \in W_{1}^{k}, \quad A_{+} \in L^{3}, \dot{A}_{+} \in L^{3 / 2} .
$$

Then $A_{0}$ satisfies the estimates

$$
\left\{\begin{array}{l}
\left\|A_{0}(t) ; W_{\infty}^{k}\right\| \leq a_{0} t^{-1} \\
\left\|\partial_{t} A_{0}(t) ; W_{\infty}^{k-1}\right\| \leq a_{0} t^{-1} \quad \text { for } k \geq 1 .
\end{array}\right.
$$

A proof can be found in [20]. As mentioned in Remark 1.2, the assumptions $A_{+} \in L^{3}$ and $\dot{A}_{+} \in L^{3 / 2}$ serve to exclude constants in $A_{+}$and $\dot{A}_{+}$and linear terms in $x$ in $A_{+}$, but are otherwise controlled by the $W_{1}^{k}$ assumption through Sobolev inequalities.

We next derive some preliminary estimates of $\widetilde{A}_{1}$ and $\widetilde{\widetilde{A}}_{1}$.

Lemma 3.4. Let $k \geq 0$ be an integer. Then the following estimates hold.

$$
\begin{aligned}
\left\|\omega^{k+1} \widetilde{A}_{1}\right\|_{2} \vee\left\|\omega^{k} \widetilde{\widetilde{A}}_{1}\right\|_{2} \leq & (k+1 / 2)^{-1}\left\|\omega^{k} x\left|w_{+}\right|^{2}\right\|_{2} \\
\left\|\omega^{k+1}\left(x \cdot \widetilde{A}_{1}\right)\right\|_{2} \leq & (k-1 / 2)^{-1}\left(\left\|\omega^{k} x^{2}\left|w_{+}\right|^{2}\right\|_{2}\right. \\
& \left.+2\left\|\omega^{k} x\left|w_{+}\right|^{2}\right\|_{2}\right) \quad \text { for } k \geq 1, \\
\left\|\nabla^{k} \widetilde{A}_{1}\right\|_{\infty} \leq & C\left\|\omega^{k} x\left|w_{+}\right|^{2} ; H^{1}\right\|, \\
\left\|\nabla^{k-1} \widetilde{\widetilde{A}}_{1}\right\|_{\infty} \leq & C\left\|\omega^{k} x\left|w_{+}\right|^{2} ; H^{1}\right\| \quad \text { for } k \geq 1 \\
\left\|\nabla^{k}\left(x \cdot \widetilde{A}_{1}\right)\right\|_{\infty} \leq & C\left(\left\|\omega^{k} x^{2}\left|w_{+}\right|^{2} ; H^{1}\right\|\right. \\
& \left.+\left\|\omega^{k} x\left|w_{+}\right|^{2} ; H^{1}\right\|\right) \quad \text { for } k \geq 1 .
\end{aligned}
$$

Proof. (3.31) follows immediately from (1.14) and (3.3). From (1.14) and from the commutation relation

$$
[x ; P]=-2 \omega^{-2} \nabla
$$

it follows that

$$
x \cdot \widetilde{A}_{1}=\int_{1}^{\infty} d \nu \nu^{-2} \omega^{-1} \sin (\omega(\nu-1)) D_{0}(\nu)\left\{P \cdot(x \otimes x)\left|w_{+}\right|^{2}-2 \omega^{-2} \nabla \cdot x\left|w_{+}\right|^{2}\right\}
$$


so that

$$
\begin{aligned}
\left\|\omega^{k+1} x \cdot \widetilde{A}_{1}\right\|_{2} \leq & \int_{1}^{\infty} d \nu \nu^{-2}\left(\left\|\omega^{k} D_{0}(\nu) x^{2}\left|w_{+}\right|^{2}\right\|_{2}\right. \\
& \left.+2(\nu-1)\left\|\omega^{k+1} D_{0}(\nu) \omega^{-1} x\left|w_{+}\right|^{2}\right\|_{2}\right) \\
\leq & \int_{1}^{\infty} d \nu \nu^{-1 / 2-k}\left(\left\|\omega^{k} x^{2}\left|w_{+}\right|^{2}\right\|_{2}+2\left\|\omega^{k} x\left|w_{+}\right|^{2}\right\|_{2}\right)
\end{aligned}
$$

which implies (3.32). Finally (3.33)-(3.35) follow from (3.31) (3.32) and from the fact that $\dot{H}^{1} \cap \dot{H}^{2} \subset L^{\infty}$.

As an immediate corollary, we obtain the following estimates of $A_{1}$ and $\partial_{t} A_{1}$.

Corollary 3.1. The following estimates hold.

$$
\begin{aligned}
& \left\|A_{1}(t)\right\|_{\infty} \leq C\left\|w_{+} ; H^{2,1}\right\|^{2} t^{-1}, \\
& \left\|\partial_{t} A_{1}(t)\right\|_{\infty} \vee\left\|\nabla A_{1}(t)\right\|_{\infty} \leq C\left\|w_{+} ; H^{2,1}\right\|^{2} t^{-2} .
\end{aligned}
$$

Proof. The result follows from (1.13) (3.2) (3.4) and from (3.33) (3.34).

We next derive the remaining estimates of $u_{a}$ and of $R_{1,1}$. The following proposition is slightly stronger than needed.

Proposition 3.1. $\quad$ Let $u_{a}$ be defined by (1.8) with $w_{+}=F u_{+}$and with $\varphi$ defined by (1.16) (1.2) (1.14) and let $R_{1,1}$ be given by (3.28). Let $u_{+} \in$ $H^{3,1} \cap H^{1,3}$. Then the following estimates hold for some constants $c, c_{1}$ and $r_{1,1}$, for $0 \leq j+k \leq 1$ and for all $t \geq 1$ :

$$
\left\|\partial_{t}^{j} \nabla^{k} u_{a}(t)\right\|_{r} \leq c t^{-\delta(r)} \quad \text { for } 2 \leq r \leq \infty .
$$

In particular

$$
\begin{aligned}
& \left\|u_{a}(t)\right\|_{3} \leq\left\|w_{+}\right\|_{3} t^{-1 / 2}, \\
& \left\|\nabla u_{a}(t)\right\|_{4} \leq\left(\left\|x w_{+}\right\|_{4}+O\left(t^{-1} \ln t\right)\right) t^{-3 / 4} . \\
& \left\|\partial_{t}^{j} \nabla^{k+1} u_{a}(t)\right\|_{r} \leq c t^{-\delta(r)} \quad \text { for } 2 \leq r \leq 6, \\
& \left\|\partial_{t}^{j} \nabla^{k} J u_{a}(t)\right\|_{r} \leq c_{1}(1+\ell n t) t^{-\delta(r)} \quad \text { for } 2 \leq r \leq 6 \text {, } \\
& \left\|\partial_{t}^{j} \nabla^{k} R_{1,1}(t)\right\|_{2} \leq r_{1,1} t^{-2}(1+\ell n t)^{2} \text {. }
\end{aligned}
$$


Proof. From the commutation relations (3.23) (3.24), it follows that for any differential operator $Z$

$$
\left\|\partial_{t}^{j} \nabla^{k} M D Z \exp (-i \varphi) w_{+}\right\|_{r}=t^{-\delta(r)}\left\|\widetilde{\partial}_{t}^{j} \widetilde{\nabla}^{k} Z \exp (-i \varphi) w_{+}\right\|_{r} .
$$

From (1.8) (3.28) and from the commutation relation (3.25), it follows that in order to derive (3.39)-(3.44) we have to estimate norms of the type

$$
\left\|\widetilde{\partial}_{t}^{j} \widetilde{\nabla}^{k} Z \exp (-i \varphi) w_{+}\right\|_{r}
$$

for $0 \leq j+k \leq 1$, for suitable choices of $Z$ and $r$, and with suitable $r$ independent time behaviour. The relevant choices are

$$
\begin{array}{ll}
Z=1, & 2 \leq r \leq \infty \quad \text { for }(3.39) \\
Z=\widetilde{\nabla} \text { or } \nabla, & 2 \leq r \leq 6 \quad \text { for }(3.42)(3.43), \\
Z=t^{-2} \Delta, & r=2 \quad \text { for }(3.44) .
\end{array}
$$

Expanding $\widetilde{\partial}_{t}^{j} \widetilde{\nabla}^{k}$ according to the definitions (3.23) (3.24) and omitting the commutators of derivatives with powers of $x$ and $t$ which generate terms of lower order, we are led to estimate norms of the type $\left\|Z \exp (-i \varphi) w_{+}\right\|_{r}$ for the following choices of $Z, r$ :

$$
\begin{array}{lc}
Z=1, x, t^{-1} \nabla, x^{2}, \partial_{t}, t^{-1} x \cdot \nabla & \text { with } 2 \leq r \leq \infty \text { for }(3.39), \\
Z=x, x^{2}, t^{-1} x \nabla, x^{3}, x \partial_{t}, t^{-1} x^{2} \nabla, t^{-1} \nabla, t^{-2} \nabla^{2}, t^{-1} \partial_{t} \nabla, t^{-2} x \nabla^{2} & \\
& \text { with } 2 \leq r \leq 6 \text { for (3.42), } \\
Z=\nabla, x \nabla, t^{-1} \nabla^{2}, x^{2} \nabla, \partial_{t} \nabla, t^{-1} x \nabla^{2} & \text { with } 2 \leq r \leq 6 \text { for (3.43), } \\
Z=\Delta, x \Delta, t^{-1} \nabla \Delta, x^{2} \Delta, \partial_{t} \Delta, t^{-1} x \nabla \Delta & \text { with } r=2 \text { for (3.44), }
\end{array}
$$

where we have omitted an overall $t^{-2}$ factor in the last case.

We expand the derivatives acting on $\exp (-i \varphi) w_{+}$by the Leibnitz rule and we estimate the expressions thereby obtained by the Hölder inequality. For that purpose we need some control of $\varphi$. From Lemma 2.3 it follows easily that for $w_{+} \in H^{3}, \nabla g\left(\left|w_{+}\right|^{2}\right) \in H^{4}$ and in particular $\nabla^{k} g\left(\left|w_{+}\right|^{2}\right) \in L^{\infty}$ for $0 \leq k \leq 3$. Together with Lemma 3.4, this provides an estimate of $\left\|\partial_{t}^{j} \nabla^{k} \varphi\right\|_{r}$ for $j=0,1$, for $k=1,2$ and $r=\infty$ and for $k=3$ and $r=6$. With that information available, we apply the Hölder inequality according to the following rules:

(i) all the explicit powers of $x$ are attached to $w_{+}$. In addition, whenever there appears a factor $\partial_{t} \varphi$ (with no space derivative), one power $x$ is extracted from the $\widetilde{A}_{1}$ part of $\partial_{t} \varphi$ and attached to $w_{+}$(since $\widetilde{A}_{1}$ belongs to $L^{\infty}$ but a priori $\partial_{t} \varphi$ does not). 
(ii) The $x$ amputated contribution of $\partial_{t} \varphi$ generated by rule (i) and all the factors $\partial_{t}^{j} \nabla^{k} \varphi$ with $k=1,2$ are estimated in $L^{\infty}$. The factors $\nabla^{3} \varphi$ are estimated in $L^{6}$ (in fact in $H^{1}$ ). Such factors occur only from the $t^{-1} x^{s} \nabla \Delta$ terms in the proof of (3.44).

(iii) The previous rules generate norms of the type $\left\|x^{s} \nabla^{k} w_{+}\right\|_{r}$ for $w_{+}$. Those norms are estimated by $H^{1}$ norms of the same quantities for $2<r \leq 6$ and by $H^{2}$ norms for $6<r \leq \infty$.

(iv) The time dependence of the various terms follows from the explicit $t$ dependence of the operators $Z$ of the previous list, together with the fact that $\left\|\partial_{t}^{j} \nabla^{k} \varphi\right\|_{r}$ generates a factor $t^{-1}$ for $j=1$ and a factor $\ell$ n $t$ for $j=0$.

With the previous rules available, the proof reduces to an elementary book keeping exercise, which will be omitted. We simply remark that the dominant terms as regards $w_{+}$have $x^{3} \nabla, x^{2} \nabla^{2}$ and $x \nabla^{3}$ which are exactly controlled by the assumption $w_{+} \in H^{1,3} \cap H^{3,1}$, equivalent to the assumption $u_{+} \in H^{3,1} \cap$ $H^{1,3}$. As regards the time dependence, the dominant terms come from $x^{s} \nabla \varphi$ in the proof of (3.43) thereby generating a factor $\ell n t$, and from $x^{s} \Delta \exp (-i \varphi)$ in the proof of (3.44), generating $x^{s}|\nabla \varphi|^{2}$ and therefore a factor $(\ell n t)^{2}$.

Finally (3.40) is the special case $j=k=0, Z=1, r=3$ of (3.45), while (3.41) follows from the estimate

$$
\left\|\nabla u_{a}(t)\right\|_{4} \leq\left(\left\|x w_{+}\right\|_{4}+t^{-1}\left(\left\|\nabla w_{+}\right\|_{4}+\|\nabla \varphi\|_{\infty}\left\|w_{+}\right\|_{4}\right)\right) t^{-3 / 4} .
$$

We can now complete the proof of Proposition 1.1.

Proof of Proposition 1.1. It suffices to show that the assumptions of Proposition 2.2 are satisfied for the choice $h(t)=t^{-1}(2+\ell n t)^{2}$ made in Proposition 1.1. Now the assumptions (2.17) (2.19) follow from (3.39) (3.42) of Proposition 3.1, the assumption (2.20) follows from Lemma 3.3 and Corollary 3.1. The assumption (2.21) follows from Lemma 3.2 as regards $R_{1,2}$ and from (3.44) of Proposition 3.1 as regards $R_{1,1}$. The assumptions (3.11) of Lemma 3.1 and (3.19) of Lemma 3.2 are special cases of (3.43). The assumption (3.21) of Lemma 3.2 follows from Lemma 3.3 and from the fact that if $A_{0}$ is a solution of the free wave equation $\square A_{0}$ in the Coulomb gauge $\nabla \cdot A_{0}=0$, with initial data $\left(A_{+}, \dot{A}_{+}\right)$, then also $x \cdot A_{0}$ is a solution of the free wave equation, namely $\square\left(x \cdot A_{0}\right)=0$, with initial data $\left(x \cdot A_{+}, x \cdot \dot{A}_{+}\right)$. Finally the assumptions (2.22) (2.23) follow from Lemma 3.1. 
The smallness conditions needed for Proposition 2.2 bear on $c_{3}$ and $c_{4}$. Now from (3.40), $c_{3}=\left\|w_{+}\right\|_{3}$ while from (3.41) or (3.46)

$$
c_{4}=\left\|x w_{+}\right\|_{4}+O\left(T_{0}^{-1} \ln T_{0}\right) .
$$

Since the estimates are used only for $t \geq T$, one can replace $T_{0}$ by $T$ in that expression, and the last term can be made arbitrarily small by taking $T$ sufficiently large, so that the smallness condition of $c_{4}$ reduces to the smallness of $\left\|x w_{+}\right\|_{4}$.

Remark 3.1. The regularity assumptions on $u_{+}$or $w_{+}$could be somewhat weakened. The strongest assumptions come from the $\Delta w_{+}$term in $R_{1,1}$ and from the $x^{3}, x \nabla^{2}$ and $x^{2} \nabla$ operators $Z$ in the estimate of $\partial_{t} \nabla u_{a}$. On the one hand the $\Delta w_{+}$term in $R_{1,1}$ could be eliminated by the choice

$$
w(t)=U(1 / t)^{*} w_{+}
$$

at the expense of generating either a more complicated and less explicit $\varphi$ or additional terms in $R_{2}$. On the other hand, we have obtained on $L^{6}$ estimate of $\partial_{t} \nabla u_{a}$ whereas an $L^{4}$ estimate was sufficient. Only a minor weakening of the assumptions on $u_{+}$could be achieved along those lines, and we shall not press that point any further.

\section{References}

[1] Ginibre, J., Ozawa, T., Long range scattering for nonlinear Schrödinger and Hartree equations in space dimensions $n \geq 2$, Comm. Math. Phys., 151 (1993), 619-645.

[2] Ginibre, J., Velo, G., Scattering theory in the energy space for a class of non-linear Schrödinger equations, J. Math. Pures Appl., 64 (1985), 363-401.

[3] , Generalized Strichartz inequalities for the wave equation, J. Funct. Anal., 133 (1995), 50-68.

[4] Long range scattering and modified wave operators for the Wave-Schrödinger system, Ann. Henri Poincaré, 3 (2002), 537-612.

[5] _ Long range scattering and modified wave operators for the Wave-Schrödinger system II, Ann. Henri Poincaré, 4 (2003), 973-999.

[6] L Long range scattering and modified wave operators for the Maxwell-Schrödinger system I. The case of vanishing asymptotic magnetic field, Comm. Math. Phys., 236 (2003), 395-448.

[7] Scattering theory for the Schrödinger equation in some external time dependent magnetic fields, J. Differential Equations, 215 (2005), 108-177.

[8] L Long range scattering for the Wave-Schrödinger system with large wave data and small Schrödinger data, Hokkaido Math. J., in press.

[9] Guo, Y., Nakamitsu, K., Strauss, W., Global finite energy solutions of the MaxwellSchrödinger system, Comm. Math. Phys., 170 (1995), 181-196. 
[10] Keel, M., Tao, T., Endpoint Strichartz estimates, Amer. J. Math., 120 (1998), 955-980

[11] Nakamitsu, K., Tsutsumi, M., The Cauchy problem for the coupled Maxwell-Schrödinger equations, J. Math. Phys., 27 (1986), 211-216.

[12] Nakamura, M., Wada, T., Local wellposedness for the Maxwell-Schrödinger equations, Math. Ann., 332 (2005), 565-604.

[13] Ozawa, T., Long range scattering for nonlinear Schrödinger equations in one space dimension, Comm. Math. Phys., 139 (1991), 479-493.

[14] Ozawa, T., Tsutsumi, Y., Asymptotic behaviour of solutions for the coupled KleinGordon-Schrödinger equations, in Spectral and Scattering Theory and Applications, Adv. Stud. Pure Math., 23 (1994), 295-305.

[15] Shimomura, A., Wave operators for the coupled Klein-Gordon-Schrödinger equations in two space dimensions, Funkcial. Ekvac., 47 (2004), 63-82.

[16] Scattering theory for the coupled Klein-Gordon-Schrödinger equations in two space dimensions, J. Math. Sci. Univ. Tokyo, 10 (2003), 661-685.

[17] Scattering theory for the coupled Klein-Gordon-Schrödinger equations in two space dimensions II, Hokkaido Math. J., 34 (2005), 405-433.

[18] - Modified wave operators for the coupled Wave-Schrödinger equations in three space dimensions, Discrete Contin. Dyn. Syst., 9 (2003), 1571-1586.

[19] Modified wave operators for Maxwell-Schrödinger equations in three space dimensions, Ann. Henri Poincaré, 4 (2003), 661-683.

[20] Strauss, W., Non linear Wave equations, CMBS Lecture Notes 73, Amer. Math. Soc. Providence 1989.

[21] Tsutsumi, Y., Global existence and asymptotic behaviour for the Maxwell-Schrödinger system in three space dimensions, Comm. Math. Phys., 151 (1993), 543-576.

[22] Yajima, K., Existence of solutions for Schrödinger evolution equations, Comm. Math. Phys., 110 (1987), 415-426. 